San Jose State University

SJSU ScholarWorks

Master's Theses

Master's Theses and Graduate Research

Summer 2010

\title{
An Iterated Forcing Extension In Which All Aleph-1 Dense Sets of Reals Are Isomorphic
}

Michael Haig Vartanian

San Jose State University

Follow this and additional works at: https://scholarworks.sjsu.edu/etd_theses

\section{Recommended Citation}

Vartanian, Michael Haig, "An Iterated Forcing Extension In Which All Aleph-1 Dense Sets of Reals Are Isomorphic" (2010). Master's Theses. 3834.

DOI: https://doi.org/10.31979/etd.dem8-u6bu

https://scholarworks.sjsu.edu/etd_theses/3834

This Thesis is brought to you for free and open access by the Master's Theses and Graduate Research at SJSU ScholarWorks. It has been accepted for inclusion in Master's Theses by an authorized administrator of SJSU ScholarWorks. For more information, please contact scholarworks@sjsu.edu. 


\title{
AN ITERATED FORCING EXTENSION IN WHICH ALL $\aleph_{1}$-DENSE SETS OF REALS ARE ISOMORPHIC
}

\author{
A Thesis \\ Presented to \\ The Faculty of the Department of Mathematics \\ San José State University
}

In Partial Fulfillment

of the Requirements for the Degree

Master of Science

by

Michael H. Vartanian

August 2010 
(c) 2010

Michael H. Vartanian

ALL RIGHTS RESERVED 
The Designated Thesis Committee Approves the Thesis Titled

AN ITERATED FORCING EXTENSION IN WHICH ALL $\aleph_{1}$-DENSE SETS OF REALS ARE ISOMORPHIC

by

Michael H. Vartanian

APPROVED FOR THE DEPARTMENT OF MATHEMATICS

SAN JOSÉ STATE UNIVERSITY

August 2010

Dr. Maurice C. Stanley Department of Mathematics

Dr. Michael J. Beeson Department of Mathematics

Dr. Kenneth R. Kellum Department of Mathematics 


\section{ABSTRACT \\ AN ITERATED FORCING EXTENSION IN WHICH ALL $\aleph_{1}$-DENSE SETS OF REALS ARE ISOMORPHIC}

by Michael H. Vartanian

If $\kappa$ is an infinite cardinal, then $X \subseteq \mathbb{R}$ is called $\kappa$-dense if between any $a<b$, both in $\mathbb{R}$, there are exactly $\kappa$ elements of $X$. In these terms, a famous result of Cantor says that in every model of set theory all $\aleph_{0}$-dense sets of reals are isomorphic (to $\mathbb{Q}$ ). This result cannot be directly extended, however, since for $\kappa=\aleph_{1}$ there exist models of set theory in which not all $\aleph_{1}$-dense sets are isomorphic. On the other hand, Baumgartner has shown by the method of iterated forcing that assuming the consistency of set theory, there does exist at least one model of set theory in which all $\aleph_{1}$-dense subsets are isomorphic. We present here a detailed, yet expository, account of Baumgartner's result and discuss its relevance to the Proper Forcing Axiom of contemporary set theory. 


\section{TABLE OF CONTENTS}

\section{CHAPTER}

1 INTRODUCTION 1

1.1 Main problem ..................... . . 1

1.2 Overview of this work . . . . . . . . . . . . . . 3

2 SIMPLE FORCING 5

2.1 Set theory conventions . . . . . . . . . . . . . . 5

2.2 Cardinality lemmas . . . . . . . . . . . . . . . 6

$2.3 \aleph_{1}$-dense sets of reals . . . . . . . . . . . . . . . 6

2.4 Forcing . . . . . . . . . . . . . . . . . 7

2.4.1 Ground model . . . . . . . . . . . . . . . . . . . 7

2.4.2 Forcing apparatus . . . . . . . . . . . . . . . 8

2.4.3 Generic isomorphisms . . . . . . . . . . . . . . 11

2.5 C.c.c. and nice names . . . . . . . . . . . . . . . . . 12

2.5.1 Countable chain condition . . . . . . . . . . . . . 13

2.5.2 Nice names for subsets . . . . . . . . . . . . . . . . 13

3 PARTIAL ORDER $\mathbb{P}(A B) \quad 16$

3.1 Partitions of $\aleph_{1}$-dense sets . . . . . . . . . . . . . . . . 16

3.2 Back-and-forth method . . . . . . . . . . . . . . . . 18 
3.3 Diagonalization method . . . . . . . . . . . . . . . . . 19

3.3 .1 Topology . . . . . . . . . . . . . . . . . . . 20

3.3 .2 Enumeration . . . . . . . . . . . . . . . . . . 21

3.3 .3 Closure . . . . . . . . . . . . . . . . . . . . . . . . . 21

3.3 .4 Exclusion . . . . . . . . . . . . . . . . . . . . . 22

3.4 Definition of $\mathbb{P}(A B) \ldots \ldots \ldots \ldots \ldots \ldots \ldots \ldots$

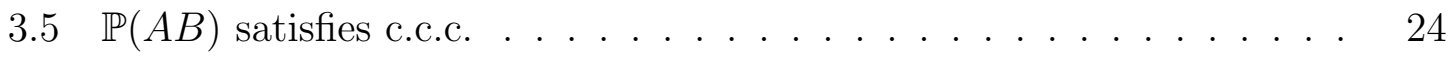

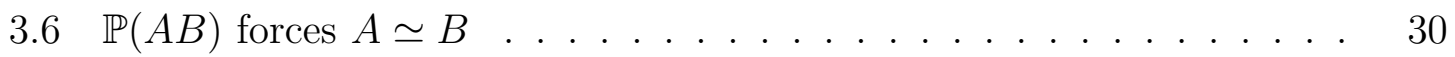

4 ITERATED FORCING 32

4.1 Overview . . . . . . . . . . . . . . . . . . . . . . . 32

4.2 Complete embeddings . . . . . . . . . . . . . . . 33

4.3 Appearance at intermediate stages . . . . . . . . . . . 35

4.4 Bookkeeping function . . . . . . . . . . . . . 36

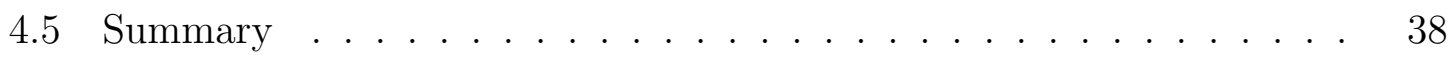

$\begin{array}{lll}5 & \text { CONCLUSION } & 39\end{array}$

$\begin{array}{ll}\text { BIBLIOGRAPHY } & 41\end{array}$ 


\section{CHAPTER 1}

\section{INTRODUCTION}

\section{$1.1 \quad$ Main problem}

Let $\mathbb{R}$ be the continuum, expressed as the ordered set of reals, and let $\aleph_{0}$ be the cardinality of the naturals. We begin with two theorems by Cantor. ${ }^{1}$ The first tells us the cardinality of $\mathbb{R}$ is greater than $\aleph_{0}$, so there is a least cardinal $\aleph_{1}>\aleph_{0}$. The second is:

There is a $\mathbb{Q}_{0} \subseteq \mathbb{R}$ such that between every two elements of $\mathbb{R}$ there are exactly $\aleph_{0}$ elements of $\mathbb{Q}_{0}$, and all $X \subseteq \mathbb{R}$ with this property are order-isomorphic to $\mathbb{Q}_{0}$.

For the property mentioned, we say that $\mathbb{Q}_{0}$ is $\aleph_{0}$-dense. Probably the simplest question one can ask is: What happens when $\aleph_{1}$ is substituted for $\aleph_{0}$ in the displayed statement? More exactly: Is there, or at least could there be, a $\mathbb{Q}_{1} \subseteq \mathbb{R}$ which, substituted for $\mathbb{Q}_{0}$, preserves the theorem? Should there be?

If we assume that our set-theoretic universe is described by only the Zermelo-Fraenkel axioms with Choice (ZFC), then there is no way to know whether such a $\mathbb{Q}_{1}$ actually exists or not. In fact, Sierpinski (c. 1950) showed that any model of set theory in which the Continuum Hypothesis $(\mathrm{CH})$ is true contains at least $\aleph_{1}$

\footnotetext{
${ }^{1}$ See [Jech03].
} 
pairwise non-isomorphic $\aleph_{1}$-dense sets. On the other hand, Baumgartner, using the modern method of forcing, established the following result, sufficient for $\mathbb{Q}_{1}[$ Baum73]:

(*) If any model of set theory exists, then a model of set theory (and $\neg \mathrm{CH}$ ) exists in which all $\aleph_{1}$-dense sets are order-isomorphic.

Thus, there could be a $\mathbb{Q}_{1}$. From these brief remarks, the place of $(*)$ in the high tradition of set theory should already be clear, and for this reason a full exposition, unavailable elsewhere, is the subject here. We also wish to comment on the importance of $(*)$ for certain recent developments in set theory, but the reader who is not particularly interested in these may now skip to the next section.

As to whether there should be a $\mathbb{Q}_{1}$, Baumgartner subsequently proposed (*), as well as several other interesting results, as evidence for adopting the Proper Forcing Axiom (PFA) as a strengthening of Martin's Axiom( $\left.\aleph_{1}\right)$ in set theory [Baum84]. This initiative came about after Shelah's introduction of proper forcing (c. 1978) by which he was able to give a simpler, albeit more sophisticated, proof of $(*)$.

One such recent result (c. 2006) concerns the basis problem for the uncountable linear orders. We say that a subclass $\mathcal{Y}$ of a class $\mathcal{X}$ of linear orders is a basis for $\mathcal{X}$ if every member of $\mathcal{X}$ contains an isomorphic copy of some element of $\mathcal{Y}$. Assuming PFA and building on key combinatorial lemmas by Todorčević [Tod98], J. T. Moore produced a five-element basis for the uncountable linear orders [Mo06]. This result actually incorporates a corollary of $(*)$ noted by Baumgartner even before the advent of PFA [Baum73]. Call a linear order real-type if it is isomorphic to an uncountable subset of $\mathbb{R}$. Then $(*)$ implies that 
$\left\{\mathbb{Q}_{1}\right\}$ is a basis for the real-type linear orders.

Moore's main result is his proof of Shelah's Conjecture that, under PFA, there exists a two-element basis for the class of uncountable linear orders whose type is neither real, $\omega_{1}$ nor $\omega_{1}^{*}$. We shall not discuss this further, except for the personal comment that an understanding of $(*)$ and its proof is required for a full appreciation and confident application of PFA itself.

\subsection{Overview of this work}

Our objective here is to present a full, and relatively complete, proof of Baumgartner's result $(*)$. In Chapter 2, we give our set-theoretic conventions and a brief introduction to the theory of simple (as opposed to iterated) forcing. We follow the development of forcing as given in the text by Kunen [Kun80]. The natural endpoint of this discussion is the existence of a ground model of set theory $M$ over which, for any fixed $\aleph_{1}$-dense sets $A, B \in M$, another model of set theory (a generic extension of $M$ ) can be forced in which $A$ and $B$ are isomorphic. However, in order to motivate the construction of a countable chain condition (c.c.c.) partial order that follows in Chapter 3, a final section is appended covering both the c.c.c. and the related topic of nice names for subsets.

Chapter 3 addresses the hard part of the problem, and for this we follow [Baum73] but with much added detail. Assuming that the ground model $M$ also satisfies $\mathrm{CH}$, we construct for any fixed $\aleph_{1}$-dense sets $A, B \in M$ a c.c.c. partial order $\mathbb{P}(A B)$ by which is forced another model in which $A$ and $B$ are isomorphic.

Chapter 4 presents a general discussion of iterated forcing by which the forcings by individual $\mathbb{P}(A B)$ 's are exhaustively iterated to finally produce a model $M^{*}$ for which $(*)$ holds. Due to the technicalities associated with the method of 
forcing, a complete and rigorous presentation of this subject is beyond the scope of this thesis; still, it is worth noting that [Baum73] devotes just one paragraph to iterated forcing, and the reader is referred instead to the now somewhat dated paper by Solovay and Tennenbaum (1971) on this subject. For our presentation, we have adapted for our purposes Kunen's more modern proof by iterated forcing of the consistency of Martin's Axiom [Kun80].

In Chapter 5, we conclude by discussing how use of the Proper Forcing Axiom simplifies the proof of Baumgartner's result. 


\section{CHAPTER 2}

\section{SIMPLE FORCING}

Our main purpose in this chapter is to establish that assuming there is any model of set theory at all, then there is a model $M$ of set theory with the property that for any given pair of $\aleph_{1}$-dense sets $A, B \in M$, there exists by the method of forcing yet another model of set theory in which $A$ and $B$ are isomorphic. Of course, this does not guarantee a model of set theory in which all $\aleph_{1}$-dense sets are isomorphic. As a first step towards this latter goal, however, we introduce in the last section the notions of countable chain condition for partial orders and nice names for subsets in generic extensions.

\section{$2.1 \quad$ Set theory conventions}

"Set theory" here means Zermelo-Fraenkel set theory with Choice (ZFC), which we assume consistent. The letters $\alpha, \beta, \gamma$ denote ordinals, $\kappa, \lambda$ cardinals, and $0=\varnothing$, the empty set. $\kappa \times \kappa$ is the set of all pairs $\langle\alpha, \beta\rangle$ with $\alpha, \beta<\kappa$. If $S$ is a set, $|S|$ denotes the cardinality of S. $\aleph_{0}$ is the least infinite cardinal; a set $A$ is countable iff $|A| \leq \aleph_{0}$, and uncountable otherwise. $\aleph_{1}$ is the least uncountable cardinal, and $\aleph_{2}$ is the least cardinal greater than $\aleph_{1}$. We use $\omega=\omega_{0}$, and $\omega_{1}, \omega_{2}$ to denote the corresponding orderings, i.e., $\omega=\left\langle\aleph_{0},<\right\rangle$, etc. The Continuum Hypothesis $2^{\aleph_{0}}=\aleph_{1}$ is denoted $\mathrm{CH}$, and the statement $2^{\aleph_{1}}=\aleph_{2}$ by $\mathrm{CH}_{1} \cdot[A]^{\kappa}$ is the collection of subsets 
of $A$ of cardinality $\kappa$.

The method of forcing was originally discovered by P. Cohen (1963), and much of the subsequent development in the 1960's is due to R. Solovay. We follow in general the development of forcing with arbitrary partial orders as given in $\left[\right.$ Kun80]. ${ }^{1}$

\subsection{Cardinality lemmas}

We state for reference the following results which will be basic for Chapters 3 and 4 (see [Kun80]):

Lemma 2.2.1. Let $\kappa$ be an infinite cardinal. Then a union of $\leq \kappa$ sets each of cardinality $\leq \kappa$ has cardinality $\leq \kappa$.

In the sequel, we refer to this consequence of the Axiom of Choice as the pigeonhole principle.

Lemma 2.2.2. If $\kappa$ is an infinite cardinal, and $n>1$ a natural number, then there exists a function $f: \kappa \rightarrow \kappa \times \cdots \times \kappa$ ( $n$ times) that is $1-1$ and onto.

This follows from the Gödel well-ordering on $\kappa \times \kappa$.

\section{$2.3 \aleph_{1}$-dense sets of reals}

A linear order is a pair $\left\langle A,<_{A}\right\rangle$, where $<_{A}$ is a subset of $A \times A$ that is an irreflexive, transitive, and total relation. Where it will not cause confusion, we often refer to $\left\langle A,<_{A}\right\rangle$ as simply $A$. If $A$ and $B$ are linear orders, then a (linear order-) isomorphism from $A$ to $B$ is a function $f: A \rightarrow B$ that is 1-1, onto, and preserves the order relation, i.e., $\forall a, a^{\prime} \in A\left(a<_{A} a^{\prime} \leftrightarrow f(a)<_{B} f\left(a^{\prime}\right)\right)$. An isomorphism $f: A \rightarrow B$ will often be abbreviated $f: A \simeq B$ below.

\footnotetext{
${ }^{1}$ [Jech03] presents an alternative development of forcing using complete Boolean algebras.
} 
Definition 2.3.1. Let $A=\left\langle A,<_{A}\right\rangle$ be a linear order with $A \subseteq \mathbb{R}$ and where $<_{A}$ is a subset of $(A \times A) \cap<_{\mathbb{R}}$ with $<_{\mathbb{R}}$ the standard order on $\mathbb{R}$. Then $A$ is $\aleph_{1}$-dense iff for all $a, b \in \mathbb{R},|(a, b) \cap A|=\aleph_{1}$.

Lemma 2.3.2. If $A$ is $\aleph_{1}$-dense, then $|A|=\aleph_{1}$.

Proof. $\mathbb{R}$, being separable, is the union of countably many open rational intervals $\left(q_{n}, r_{n}\right), n<\omega$. Thus, applying Lemma 2.2.1 with $\kappa=\aleph_{1}$ gives:

$$
|A|=|A \cap \mathbb{R}|=\left|A \cap \bigcup_{n<\omega}\left(q_{n}, r_{n}\right)\right|=\left|\bigcup_{n<\omega}\left[\left(q_{n}, r_{n}\right) \cap A\right]\right|=\aleph_{1} .
$$

As a corollary, we remark that every $\aleph_{1}$-dense set $\left\langle A,<_{A}\right\rangle$ is isomorphic to a linear order $\left\langle\aleph_{1}, \prec_{A}\right\rangle$, where $\prec_{A}$ is the image of $<_{A}$ under $f \times f$ with $f: A \rightarrow \aleph_{1}$ a bijection as guaranteed by the Lemma. The study of isomorphisms between $\aleph_{1}$-dense sets may thus be reduced to the study of isomorphisms between certain orderings of $\aleph_{1}$. We will return to this topic in Chapter 4.

\subsection{Forcing}

\subsubsection{Ground model}

We work in $\mathbf{V}$, the universe of well-founded sets. If $\Phi$ is a set of sentences and $M$ a structure in the language of set theory, then $M \models \Phi$ means that every sentence in $\Phi$ is true in $M$. Unless stated otherwise, all theorems and lemmas below are deductions from ZFC alone and thus hold in any model of ZFC. ${ }^{2}$

The method of forcing assumes a suitable ground model $M$ of ZFC (and possibly other axioms) over which is forced a generic extension of $M$, also a model of ZFC. Although the simple application of forcing discussed in this Chapter only

\footnotetext{
${ }^{2}$ However, a set defined in $\mathbf{V}$ by a formula $\phi$ may not be the same as the set defined by $\phi$ in an inner model $M$; we avoid a discussion of absoluteness.
} 
requires a countable, transitive model of $\mathrm{ZFC}^{3}$ as the ground model, in Chapter 3 we will need a model in which $\mathrm{CH}$ also holds, and in Chapter 4, one in which both $\mathrm{CH}$ and $\mathrm{CH}_{1}$ also hold. We will therefore remark on the existence of a suitable ground model $M$ of $\mathrm{ZFC}+\mathrm{CH}+\mathrm{CH}_{1}$.

Lemma 2.4.1. Assume that $\phi_{1}, \ldots, \phi_{n}$ are axioms of ZFC. Then there exists a model $M$ such that $|M|=\aleph_{0}, M$ is transitive, and $M \models \bigwedge_{i=1}^{n} \phi_{i} \wedge C H \wedge C H_{1}$.

Remark. Gödel's constructible universe $\mathbf{L}$ satisfies the axioms, and applying the Skolem-Lowenheim Theorem and the Mostowski Collapsing Theorem gives a countable transitive model $M$ of the same. See [Kun80].

\subsubsection{Forcing apparatus}

We now fix an $M$ as given by the previous Lemma, and a pair $A, B$ of $\aleph_{1}$-dense sets in $M$. By forcing over a certain partial order $\mathbb{P} \in M$, we can extend $M$ to a generic extension $M[G]$ in which $A \simeq B$. These terms are defined as follows.

Definition 2.4.2. A partial order is a triple $\left\langle\mathbb{P}, \leq, 1_{\mathbb{P}}\right\rangle$ such that (i) $\mathbb{P} \neq 0$; (ii) $\leq$ is a reflexive and transitive relation on $\mathbb{P}$; (iii) $1_{\mathbb{P}}$ is a largest element of $\mathbb{P}$ under $\leq$. If $p \leq q$, we say that $p$ extends $q . D \subseteq \mathbb{P}$ is dense in $\mathbb{P}$ iff $\forall p \in \mathbb{P} \exists q \in D(q \leq p)$.

Definition 2.4.3. Let $M$ be a transitive model of $\mathrm{ZFC}$ and $\mathbb{P} \in M$ a partial order. $G \subseteq \mathbb{P}$ is a filter on $\mathbb{P}$ iff (i) $\forall p, q \in G \exists r \in G(r \leq p \wedge r \leq q)$ and (ii) $\forall p \in G \forall q \in \mathbb{P}(p \leq q \rightarrow q \in G)$. Furthermore, $G$ is $\mathbb{P}$-generic over $M$ iff $G$ is a filter on $\mathbb{P}$ and for all dense $D \in M, G \cap D \neq 0$.

An important fact is:

\footnotetext{
${ }^{3}$ More precisely, a finite fragment of ZFC.
} 
Lemma 2.4.4. If $M$ is a countable transitive model of $Z F C$ and $\mathbb{P} \in M$ is a partial order, then there exists a $G$ which is $\mathbb{P}$-generic over $M$.

Remark. $G$ is constructed by induction on $\omega$ using a countable enumeration of the dense sets in $M$.

Now let $\mathbb{P}$ be a partial order in $M$. A generic extension by $\mathbb{P}$ over $M$ is constructed by first defining in $\mathbf{V}$ the class of $\mathbb{P}$-names. A $\mathbb{P}$-name $\tau$ is any relation satisfying the recursive condition

$$
\text { For all }\langle\sigma, p\rangle \in \tau, \sigma \text { is a } \mathbb{P} \text {-name and } p \in \mathbb{P} \text {, }
$$

but in practice we only consider the set $M^{\mathbb{P}}$ of $\mathbb{P}$-names in $M$. Now fix a $G \mathbb{P}$-generic over $M$. If $\tau \in M^{\mathbb{P}}$, then $\tau_{G}$, the value of $\tau$ under $G$, is defined recursively by:

$$
\tau_{G}=\left\{\sigma_{G}: \exists p \in G\langle\sigma, p\rangle \in \tau\right\}
$$

and the generic extension $M[G]$ of $M$ by $G$ by:

$$
M[G]=\left\{\tau_{G}: \tau \text { is a } \mathbb{P} \text {-name in } M\right\}
$$

It may be shown that $M \subseteq M[G]$ (so $M[G]$ is indeed an extension of $M$ ), and that also $G \in M[G]$. In order to show that $M[G]$ is a model of ZFC, one defines the forcing relation $p \Vdash \phi$ between elements $p \in \mathbb{P}$ and sentences $\phi$ involving $\mathbb{P}$-names as follows:

$$
\begin{aligned}
p \Vdash \phi\left(\tau_{1}, \ldots, \tau_{n}\right) \quad \text { iff } \quad \forall G[(G \text { is } \mathbb{P} \text {-generic over } M & \wedge p \in G) \Longrightarrow \\
& \left.M[G]=\phi\left(\tau_{1 G}, \ldots, \tau_{n G}\right)\right] .
\end{aligned}
$$

In words, $p \Vdash \phi$ exactly when $\phi\left(\tau_{1_{G}}, \ldots, \tau_{n_{G}}\right)$ is satisfied in every generic extension $M[G]$ for which $G$ contains $p$.

A crucial property of $\Vdash$ is that although this relation is defined in $\mathbf{V}$, it is equivalent to a relation definable in $M$; by this means, therefore, one can define 
$\mathbb{P}$-names in $M$ whose images in $M[G]$ have desired properties. This forms part of the Forcing Theorem:

Theorem 2.4.5. Let $\phi\left(x_{1}, \ldots, x_{n}\right)$ be a formula in the language of set theory. Then for all $G \mathbb{P}$-generic over $M$,

$$
M[G] \models \phi\left(\tau_{1 G}, \ldots, \tau_{n G}\right) \quad \text { iff } \quad \exists p \in G\left(p \Vdash \phi\left(\tau_{1}, \ldots, \tau_{n}\right)\right) .
$$

We note in passing that since $1_{\mathbb{P}}$, the greatest element in $\mathbb{P}$, belongs to every generic $G$, the relation

$$
1_{\mathbb{P}} \Vdash \phi\left(\tau_{1}, \ldots, \tau_{n}\right)
$$

means that $\phi\left(\tau_{1_{G}}, \ldots, \tau_{n_{G}}\right)$ is satisfied in all generic extensions of $M$ by $\mathbb{P}$.

Using the Forcing Theorem, one can now prove:

Theorem 2.4.6. If $M$ is a countable transitive model of ZFC, $\mathbb{P}$ a partial order in $M$, and $G \mathbb{P}$-generic over $M$, then $M[G]$ is also a countable transitive model of $Z F C$.

Remark. Besides Extensionality, which is true in any transitive model, the axioms of ZFC assert that, given certain sets (and possibly formulas), there exist certain other sets (e.g., given $a$ and $b$, there is the pair $\{a, b\})$. Since $M[G]=\left\{\tau_{G}: \tau \in M^{\mathbb{P}}\right\}$, the proof of the Theorem is reduced to showing that given certain $\mathbb{P}$-names, there exists a $\mathbb{P}$-name for the desired set in $M[G]$. Generally, the fact that each axiom is already satisfied in $M$ forces its satisfaction in $M[G]$. We give the following concrete example of how the forcing relation is used to define a suitable $\mathbb{P}$-name (see [Kun80]):

Comprehension Axiom Scheme in $M[G]$. Let $M, \mathbb{P}$ and $G$ be as above. Given $\sigma_{G}$ in $M[G]$ and any formula $\phi(x)$, a name $\rho \in M^{\mathbb{P}}$ for the set of elements $a \in \sigma_{G}$ 
satisfying $\phi(a)$ in $M[G]$ is

$$
\rho=\{\langle\pi, p\rangle: \pi \in \operatorname{dom}(\sigma) \wedge p \in \mathbb{P} \wedge p \Vdash(\pi \in \sigma \wedge \phi(\pi))\}
$$

In this case, Comprehension in $M$ has already been used to show that the forcing relation is definable in $M$ (which thus gives $\rho \in M^{\mathbb{P}}$ ).

\subsubsection{Generic isomorphisms}

Suppose $A$ and $B$ are fixed $\aleph_{1}$-dense sets in $M$. To obtain an extension $M[G]$ in which $A$ is isomorphic to $B$, it is natural to consider the partial order $F n_{<}(A B)$ consisting of all finite approximations to such isomorphisms.

Definition 2.4.7. Suppose $A=\left\langle A,<_{A}\right\rangle, B=\left\langle B,<_{B}\right\rangle$ are $\aleph_{1}$-dense sets. Let

$$
\begin{aligned}
& F n_{<}(A B)=\{p: p \text { is a function } \wedge|p|<\aleph_{0} \wedge \operatorname{dom}(p) \subseteq A \\
& \wedge \forall a, a^{\prime} \in \operatorname{domg}(p) \subseteq B \wedge \\
&\left.\left.\wedge a<_{A} a^{\prime} \leftrightarrow p(a)<_{B} p\left(a^{\prime}\right)\right]\right\},
\end{aligned}
$$

and define the partial order $\left\langle F n_{<}(A B), \leq\right\rangle \in M$ by setting for all $p, q \in F n_{<}(A B)$, $p \leq q$ iff $p \supseteq q$

That $F n_{<}(A B)$ is a partial order is easily confirmed; moreover, taking $\mathbb{P}=F n_{<}(A B)$ gives the result we seek:

Theorem 2.4.8. Let $A, B \in M$ be $\aleph_{1}$-dense, and let $G$ be $F n_{<}(A B)$-generic over $M$. Then $\bigcup G$ is an isomorphism from $A$ to $B$ in $M[G]$.

Proof. Since $G \in M[G], \bigcup G \in M[G]$. We need to show that $\bigcup G$ maps $A$ onto $B$, and if $a, a^{\prime} \in A$ with $a<_{A} a^{\prime}$, then $\bigcup G(a)<_{B} \bigcup G\left(a^{\prime}\right)$. First, we claim that $D_{a}=\left\{p \in F n_{<}(A B): a \in \operatorname{dom}(p)\right.$ and $R_{b}=\left\{p \in F n_{<}(A B): b \in \operatorname{rng}(p)\right\}$ are dense in $F n_{<}(A B)$. In fact, let $q \in F n_{<}(A B)$ and assume $a \notin \operatorname{dom}(q)$. Then, since 
between every distinct $b^{\prime}, b^{\prime \prime} \in B$ there is a $b \in B$, we can adjoin a pair $\langle a, b\rangle \in A \times B$ so that $p=q \cup\{\langle a, b\rangle\}$ is order-preserving; this gives $p \in D_{a}$ and

$p \leq q$, and similarly for $R_{b}$. Since $G$ is generic, $G \cap D_{a} \neq 0 \neq G \cap R_{b}$ for all

$a \in A, b \in B$ so we have $\bigcup G: A \rightarrow B$ is onto. Moreover, if $a<_{A} a^{\prime}$, there is a

$p \in G$ with $a, a^{\prime} \in \operatorname{dom}(p)$, so $p(a)<_{B} p\left(a^{\prime}\right)$, giving $\bigcup G(a)<_{B} \bigcup G\left(a^{\prime}\right)$.

Remarks. Note that we do not claim that $A$ and $B$ are $\aleph_{1}$-dense in $M[G]$, and that the proof only requires that $A$ and $B$ be dense in $\mathbb{R}$ (rather than $\aleph_{1}$-dense).

\section{$2.5 \quad$ C.c.c. and nice names}

We have just seen that there is a model of set theory $M$ with the property that for any $A, B \aleph_{1}$-dense in $M$, there exists a generic extension $M[G]$ in which $A, B$ are isomorphic. However, nothing guarantees that $A, B$ are $\aleph_{1}$-dense in $M[G]$, so the forcing we used, $F n_{<}(A B)$, does not get us far towards our goal of a model in which all such sets are isomorphic. Moreover, even if $A, B$ were $\aleph_{1}$-dense in $M[G]$, we must still exhaust all such pairs by (presumably) iterating the above construction in some manner; but since new $\aleph_{1}$-dense sets may appear at successive stages, this process may still not "converge" to the model we seek.

It is clear we need more control over the properties of $M[G]$, and for this, the forcing partial order must have a certain property that $F n_{<}(A B)$ does not: namely, the countable chain condition (c.c.c.). This condition not only preserves cardinals in extensions over $M$, but also gives an upper bound $\left(=\aleph_{2}\right)$ to the number of nice names for (certain copies of) $\aleph_{1}$-dense sets at any stage in the iteration. These properties will enable us to define in Chapter 4 a suitable iterated forcing structure to force the desired model. We introduce these ideas here in order to motivate our construction of a c.c.c. partial order $\mathbb{P}(A B)$ in the next Chapter. In what follows, $M$ 
denotes a fixed countable transitive model of ZFC.

\subsubsection{Countable chain condition}

Definition 2.5.1. If $\mathbb{P}$ is a partial order, then $A \subseteq \mathbb{P}$ is called an antichain in $\mathbb{P}$ iff for all $p, q \in A(p \neq q \rightarrow \neg \exists r \in \mathbb{P}$ such that $r \leq p$ and $r \leq q)$. $\mathbb{P}$ satisfies the countable chain condition (c.c.c.) iff $|A| \leq \aleph_{0}$ for all antichains $A \subseteq \mathbb{P}$.

We will need the following:

Lemma 2.5.2. Let $M$ and $\mathbb{P}$ be as above, $G \mathbb{P}$-generic over $M, A \in M, A \subseteq \mathbb{P}$. Then either

$$
G \cap A \neq 0 \quad \text { or } \quad \exists q \in G \forall p \in A(p \neq q \rightarrow \neg \exists r(r \leq p \wedge r \leq q))
$$

hence, if $A$ is a maximal antichain in $\mathbb{P}$, then $G \cap A \neq 0$.

\subsubsection{Nice names for subsets}

If $c \in M$, then we can use antichains in $\mathbb{P}$ to define a set of nice names for the subsets of $c$ in a generic extension $M[G]$. The names are "nice" because every such subset has a nice name and if $\mathbb{P}$ happens to satisfy the c.c.c., then an upper bound exists for the number of nice names. Fix $M, \mathbb{P}$, and $G$ as above.

Definition 2.5.3. For any $a \in M, \check{a}$ - the canonical $\mathbb{P}$-name of $a$ - is defined recursively by $\check{a}=\left\{\left\langle\check{b}, 1_{\mathbb{P}}\right\rangle: b \in a\right\} .4$

Definition 2.5.4. Suppose $a, \mu \in M$, where $\mu$ is $\mathbb{P}$-name. Suppose also that $A_{a}$ is a maximal antichain in $\mathbb{P}$ with the property that for all $p \in A_{a}$, either $p \Vdash \check{a} \notin \mu$ or $p \Vdash \check{a} \in \mu$. Partition $A_{a}$ by defining $A_{a}^{0}=\left\{p \in A_{a}: p \Vdash \check{a} \notin \mu\right\}$ and $A_{a}^{1}=\left\{p \in A_{a}: p \Vdash \check{a} \in \mu\right\}$.

\footnotetext{
${ }^{4}$ Clearly, $\check{a}$ is a $\mathbb{P}$-name, and $a \in M$ gives $\check{a} \in M$ by absoluteness; then by induction, $(\check{a})_{G}=a$, so in fact $M \subseteq M[G]$ as previously claimed.
} 
Definition 2.5.5. A nice $\mathbb{P}$-name is any set of the form $\tau=\bigcup\left\{\{\check{a}\} \times A_{a}^{1}: a \in c\right\}$, for some $c \in M$ and a sequence of $A_{a}^{1}$ defined for some $\mathbb{P}$-name $\mu$ as above.

It is easily seen that a nice $\mathbb{P}$-name $\tau$ is in fact a $\mathbb{P}$-name. We also have that every subset in $M[G]$ of a $c \in M$ has a nice name whose domain is a set:

Lemma 2.5.6. Suppose $\mu$ is a $\mathbb{P}$-name for a subset in $M[G]$ of $c \in M$. Then there exists a nice name $\tau$ such that $\operatorname{dom}(\tau) \subseteq\{\check{a}: a \in c\}$ and $\mu_{G}=\tau_{G}$.

Proof. By Zorn's Lemma in $M$, for all $a \in c$ there exist maximal antichains $A_{a}$ (for $\mu$ ) as assumed in Definition 2.5.4. Define $\tau$ by Definition 2.5.5; then $\operatorname{dom}(\tau) \subseteq\{\check{a}: a \in c\}$. By Lemma 2.5.2, we know that if $G$ is $\mathbb{P}$-generic over $M$, then $G \cap A_{a} \neq 0$ for all $a \in c$.

Suppose now $x \in \mu_{G}$. Then $x=a_{G}$ for some $a \in c$. Since $M[G] \models a_{G} \in \mu_{G}$, by the Forcing Theorem 2.4.5 in one direction there exists $p \in G$ such that $p \Vdash \check{a} \in \mu$. Since the elements of $G$ are compatible, $\neg \exists p \in G$ with $p \Vdash \check{a} \notin \mu$. Thus $G \cap A_{a}^{0}=0$, so that $G \cap A_{a}^{1} \neq 0$. Since $\tau_{G}=\left\{a_{G}: \exists p \in G \cap A_{a}^{1}(\langle\check{a}, p\rangle \in \tau)\right\}$, we have $x=a_{G} \in \tau_{G}$. On the other hand, suppose $x \in \tau_{G}$. Let $a \in \operatorname{dom}(\tau)$ be such that $x=a_{G}$ and $G \cap A_{a}^{1} \neq 0$. If $p \in G \cap A_{a}^{1}$, then $p \Vdash \check{a} \in \mu$. Therefore, by the Forcing Theorem in the other direction, $x=a_{G} \in \mu_{G}$.

Finally, we discuss how the c.c.c. gives an upper bound to the number of nice names for certain copies of $\aleph_{1}$-dense sets in generic extensions of $M$. In Chapter 4 , the ground model $M$ will satisfy $\mathrm{CH}_{1}$ as well as $\mathrm{CH}$; furthermore, we will be concerned with c.c.c. $\mathbb{P}$ 's of size $\aleph_{1}$ and subsets of $\aleph_{1} \times \aleph_{1}$. The following will then be applicable:

Lemma 2.5.7. Assume that $C H$ and $\mathrm{CH}_{1}$ hold in $M$, and that partial order $\mathbb{P} \in M$ is c.c.c. with $|\mathbb{P}|=\aleph_{1}$. Then for any generic $G \subseteq \mathbb{P}$, there are $\leq \aleph_{2}$ nice $\mathbb{P}$-names in 
$M$ for the subsets of $\aleph_{1} \times \aleph_{1}$ in $M[G]$.

Proof. Let $\mathcal{A}$ denote the collection of antichains of $\mathbb{P}$, and fix a generic $G$. Since each antichain is a countable subset of $\mathbb{P}$ and $|\mathbb{P}|=\aleph_{1},|\mathcal{A}| \leq\left[\aleph_{1}\right] \leq \aleph_{0}=\aleph_{1}^{\aleph_{0}}$. By Lemma 2.5.6 with $c=\aleph_{1} \times \aleph_{1}$, each nice $\mathbb{P}$-name $\tau$ for a subset of $\aleph_{1} \times \aleph_{1}$ in $M[G]$ is determined by a function $f: \operatorname{dom}(\tau) \rightarrow \mathcal{A}$ and $|\operatorname{dom}(\tau)| \leq\left|\aleph_{1} \times \aleph_{1}\right|=\aleph_{1}$. Since there are $\leq|\mathcal{A}|^{\aleph_{1}}$ such functions, the number of nice $\mathbb{P}$-names for subsets of $\aleph_{1} \times \aleph_{1}$ in $M[G]$ is at most:

$$
\left(\aleph_{1}^{\aleph_{0}}\right)^{\aleph_{1}}=\aleph_{1}^{\aleph_{1}}=2^{\aleph_{1}}=\aleph_{2} .
$$




\section{CHAPTER 3}

\section{PARTIAL ORDER $\mathbb{P}(A B)$}

\subsection{Partitions of $\aleph_{1}$-dense sets}

We now turn to the core of the problem, which is to show there is a ground model $M$ of set theory such that for any two $\aleph_{1}$-dense sets $A, B \in M$ there exists a c.c.c. partial order $\mathbb{P}(A B)$ which forces an isomorphism between $A$ and $B$ in any generic extension by $\mathbb{P}(A B)$ over $M$. As we have already discussed, and shall further see in Chapter 4, the c.c.c. property of $\mathbb{P}(A B)$ is crucial for proving (among other things) the convergence of the overall iterated forcing construction.

Let $M$ be any countable transitive model of $\mathrm{ZFC}+\mathrm{CH},{ }^{1}$ and consider again

$$
F n_{<}(A B)=\{p \in M: p \text { is a finite, order-preserving function from } A \text { to } B\}
$$

ordered by reverse inclusion. In Section 2.4 we saw that $F n_{<}(A B)$ does in fact force the desired isomorphism between $A$ and $B$ in any extension over $M$, even without CH. However, because $A$ and $B$ are uncountable, $F n_{<}(A B)$ is certainly not c.c.c., since any set of the form $\{\{\langle a, b\rangle\}: b \in B\}$ for fixed $a \in A$ is an uncountable antichain in $F n_{<}(A B)$. In fact, the elements of such antichains are incompatible even as functions, not to mention order-preserving functions.

\footnotetext{
${ }^{1}$ That such an $M$ exists was discussed in Section 2.2.
} 
On the other hand, an obvious way to avoid at least this kind of antichain is to restrict to a countable set the $b \in B$ that are paired with a fixed $a \in A$. Since this requirement should be symmetric in $A$ and $B$, one is led to the idea of partitioning of $A$ and $B$ into countable pieces and then defining a suborder $\mathbb{P}(A B)$ by restriction to those $p \in F n_{<}(A B)$ that respect this partition. This idea will be made precise below.

Of course, the partitioning must be done in such a way so that all uncountable antichains in $F n_{<}(A B)$ are eliminated, while the property that $\mathbb{P}(A B)$ forces $A$ and $B$ to be isomorphic is retained. Regarding this latter requirement, however, there is reason to hope, since the proof in Section 2.4 that $F n_{<}(A B)$ forces an isomorphism required only that $A$ and $B$ be dense linear orders. Thus, it should be possible to partition the $\aleph_{1}$-dense sets $A$ and $B$ into $\aleph_{1}$ countable pieces each dense in $A$ or $B$, and expect that the induced $\mathbb{P}(A B)$ will still force the desired isomorphism. That this is indeed the case will be proved below.

The preceding suggests that the construction of $\mathbb{P}(A B)$ will involve two distinct methods. In fact, we will first use a back-and-forth method in order to partition $A$ and $B$ into $\aleph_{1}$ countable sets $A_{\alpha}$ and $B_{\alpha}$, each of which is dense in $A$ or $B$. We may think of this method as eliminating uncountable antichains whose elements are incompatible as functions. The second is a variation of the diagonalization method that, roughly speaking, excludes from $A_{\alpha}$ and $B_{\alpha}$ any element of $A$ or $B$ that would form an element of a partial function lying in an uncountable antichain indexed by some $\beta<\alpha$. This method serves to elimininate antichains whose elements are incompatible as order-preserving functions. Although these methods will be discussed separately, they are applied simultaneously in the recursive definition of $A_{\alpha}$ and $B_{\alpha}$ below. 


\subsection{Back-and-forth method}

Let $A$ and $B$ be $\aleph_{1}$-dense sets of reals. Since $|A|,|B|=\aleph_{1}$ (Lemma 1.3.3), let $\left\langle a_{\alpha}: \alpha<\omega_{1}\right\rangle$ and $\left\langle b_{\alpha}: \alpha<\omega_{1}\right\rangle$ be enumerations of $A$ and $B$, respectively.

Furthermore, let $\left\langle r_{n}: n<\omega\right\rangle$ enumerate the rational open intervals in $\mathbb{R}$.

Lemma 3.2.1. For all $\alpha<\omega_{1}$, there exist $A_{\alpha}$ and $B_{\alpha}$ such that:

(i) $A=\bigcup\left\{A_{\alpha}: \alpha<\omega_{1}\right\}$ and $B=\bigcup\left\{B_{\alpha}: \alpha<\omega_{1}\right\}$;

(ii) $A_{\alpha} \cap A_{\beta}=0=B_{\alpha} \cap B_{\beta}$ if $\alpha \neq \beta$;

(iii) For all $\alpha<\omega_{1}, A_{\alpha}$ and $B_{\alpha}$ are countable and dense in $A$ and $B$, respectively.

Proof. We define sequences $A_{\alpha}, B_{\alpha}$ by transfinite recursion on $\alpha<\omega_{1}$ with a subrecursion on $\omega$ at each $\alpha$. Suppose $A_{\beta}$ and $B_{\beta}$ have been defined for all $\beta<\alpha$. Set

$$
A_{\alpha}=\left\{k_{\alpha 0}, \ldots, k_{\alpha n}, \ldots\right\}, \quad B_{\alpha}=\left\{l_{a 0}, \ldots, l_{\alpha n}, \ldots\right\}
$$

where the $k_{\alpha n}$ and $l_{\alpha n}$ for $n<\omega$ are defined as follows. We can always write $\alpha=\beta+m$, with $\beta$ either 0 or a limit and $m<\omega$. Call $\alpha$ even (odd) if $m$ is even (odd).

If $\alpha$ is even, then using the enumeration $\left\langle a_{\alpha}\right\rangle$ define first $k_{\alpha 0}=a_{\gamma}$ where $\gamma<\omega_{1}$ is the least index such that $a_{\gamma} \notin A_{\beta}$ for all $\beta<\alpha$. Since every $\alpha_{\gamma}$ is eventually in some $A_{\alpha}$, it is clear that $A=\bigcup A_{\alpha}$. If $\alpha$ is odd, define $l_{\alpha 0}$ in a similar manner using $\left\langle b_{\alpha}\right\rangle$; then also $B=\bigcup B_{\beta}$.

The successive $k_{\alpha n}$ and $l_{\alpha n}$ are then chosen in a back-and-forth manner. For concreteness, assume $\alpha$ is even. If $k_{\alpha n}$ for some $n$ is the last element defined, then choose $l_{\alpha n}$ so that

$$
l_{\alpha n} \in B \cap r_{n}-\bigcup\left\{B_{\beta}: \beta<\alpha\right\},
$$


and if $l_{\alpha n}$ is the last element defined, then choose $k_{\alpha n+1}$ so that

$$
k_{\alpha n+1} \in A \cap r_{n+1}-\bigcup\left\{A_{\beta}: \beta<\alpha\right\}
$$

To see that these definitions make sense, note that since $B$ is $\aleph_{1}$-dense, $\left|B \cap r_{n}\right|=\aleph_{1}$, while on the other hand $\left|\cup B_{\beta}\right|=\aleph_{0}$; thus such a $l_{\alpha n}$ exists, and similarly for $k_{\alpha n+1}$.

Furthermore, since $B \cap r_{n}$ is a basis element of the space $B$, the definition guarantees that each $B_{\alpha}$ is dense in $B$; and similarly for $A_{\alpha}$ in $A$. Finally, it is clear the definition also gives that if $\alpha \neq \beta$ then $B_{\alpha} \cap B_{\beta}=0$; similarly for $A$. Thus the $A_{\alpha}$ and $B_{\alpha}$ have properties $(i),(i i)$ and $(i i i)$.

\subsection{Diagonalization method}

The partitions of $A$ and $B$ defined above eliminate uncountable antichains of the form $\{\{\langle a, b\rangle\}: b \in B\}$ for fixed $a \in A$, but do not necessarily eliminate uncountable antichains involving incompatibility as order-preserving functions. For this, we need an additional restriction on the elements of $A$ and $B$ from which the $k_{\alpha n}$ and $l_{\alpha n}$ may be selected.

We have not yet exploited the fact that $A$ and $B$ are uncountable, separable subspaces of $\mathbb{R}$. As will be shown below, every uncountable antichain contains contains an uncountable antichain consisting of elements $p$ with $|p|=n$ for some

fixed $n$. As such, $U$ will be separable as a subspace of $\mathbb{R}^{2 n}$ and will therefore be contained in the closure of some countable dense subset. By applying $\mathrm{CH}$ these closures may be enumerated by $\left\langle c_{\alpha}: \alpha<\omega_{1}\right\rangle$.

In the second step of our definition of $A_{\alpha}$ and $B_{\alpha}$, we will then use the $c_{\beta}$ for $\beta<\alpha$ to exclude any element of $A$ or $B$ that would form a $p \in U \subseteq c_{\beta}$. Of course, in order not to upset the results obtained already in Lemma 3.2.1, we need to make sure that only countable sets are excluded. 


\subsubsection{Topology}

We now make these ideas precise. As indicated above, we need only consider those $U \subseteq F n_{<}(A B)$ such that $|p|=n$ for all $p \in U$. Since $U \subseteq(A \times B)^{n}$, and since $(A \times B)^{n}$ is essentially a subset of $\mathbb{R}^{2 n}, U$ contains a countable subset dense in the relative topology on $U$. It is therefore enough to discuss the topology on $F n_{<}(A B) \cap(A \times B)^{n}$ as a subspace of $\mathbb{R}^{2 n}$.

Let $I$ denote the set of all closed rectangles in $\mathbb{R}^{2}$ whose corners have rational coordinates. It is clear that $|I|=\aleph_{0}$. If $r, r^{\prime}, s, s^{\prime}$ are closed rational intervals in $\mathbb{R}$, then two pairs $r \times s, r^{\prime} \times s^{\prime}$ in $I$ are called separated if $r \cap r^{\prime}=0=s \cap s^{\prime}$.

Let $S$ be the collection of all finite subsets of $I$ all of whose elements are pairwise separated. If $x \in S$ and $|x|=n$, fix an enumeration $x=\left\{i_{j}: j<n\right\}$, where each $i_{j}$ is a closed rectangle in $\mathbb{R}^{2}$. We see

Lemma 3.3.1. $|S|=\aleph_{0}$.

Proof. Using the fact that $|I|=\aleph_{0}$, we have

$$
|S| \leq\left|[I]^{<\aleph_{0}}\right|=\left|\aleph_{0}^{<\aleph_{0}}\right|=\aleph_{0}
$$

on the other hand, enumerating the rational rectangles in $\mathbb{R}^{2}$ shows that $\aleph_{0} \leq|S|$.

Now for each $x \in S$, define

$$
P(x)=\left\{p \in F n_{<}(A B):|p|=|x| \text { and } p \cap i_{j}=1 \text { for each } j<n\right\} .
$$

Intuitively, $P(x)$ consists of all finite functions from $A$ to $B$ that have exactly one element $\langle a, b\rangle$ in each rectangle of $x$.

Lemma 3.3.2. For all $x \in S,|P(x)|=\aleph_{1}$.

Proof. Let $x=\left\{i_{j}: j<n\right\}$. For each $j<n,\left|i_{j} \cap(A \times B)\right|=\aleph_{1} \times \aleph_{1}=\aleph_{1}$ since $A$ and $B$ are $\aleph_{1}$-dense. It follows that $|P(x)|=\left|\aleph_{1}^{n}\right|=\aleph_{1}$. 


\subsubsection{Enumeration}

We will eventually show that, given an uncountable $U \subseteq \mathbb{P}(A B)$, it may be assumed that $U \subseteq P(x)$ for some $x \in S$. Since each such $U$ countains a dense countable subset, we seek an enumeration for the collection of countable subsets of $P(x)$ taken over all $x \in S$. Accordingly, let

$$
\mathcal{C}=\{d: \exists x \in S(d \text { is a countable subset of } P(x))\}
$$

Lemma 3.3.3. (CH) $|\mathcal{C}|=\aleph_{1}$.

Proof. First, fix $x \in S$. Since $|P(x)|=\aleph_{1}$ (Lemma 3.3.2), we claim that the collection of countable subsets of $P(x)$, i.e., $[P(x)]^{\leq \aleph_{0}}$, has cardinality $\aleph_{1}$. In fact, by $\mathrm{CH}$,

$$
\left|[P(x)]^{\leq \aleph_{0}}\right|=\left|\left[\aleph_{1}\right]^{\leq \aleph_{0}}\right| \leq\left(2^{\aleph_{0}}\right)^{\aleph_{0}}=2^{\aleph_{0}}=\aleph_{1},
$$

while the collection of singletons of elements in $P(x)$ shows that $\aleph_{1} \leq\left|[P(x)] \leq \aleph_{0}\right|$. Thus, since $|S|=\aleph_{0}$ (Lemma 3.3.1), we have

$$
|\mathcal{C}|=\left|\bigcup\left\{[P(x)]^{\leq \aleph_{0}}: x \in S\right\}\right|=\aleph_{1}
$$

With this result in hand, we now fix an enumeration $\left\langle d_{\alpha}: \alpha<\omega_{1}\right\rangle$ for $\mathcal{C}$, and a sequence $\left\langle x_{\alpha}: \alpha<\omega_{1}\right\rangle$ in $S$ such that each $d_{\alpha}$ is a countable subset of $P\left(x_{\alpha}\right)$.

\subsubsection{Closure}

Here we show that any uncountable $U \subseteq P(x)$ for $x \in S$ can be "closed off" in a following sense. For each $\alpha<\omega_{1}$, define

$$
c_{\alpha}=\left\{p \in F n_{<}(A B): \forall x \in S\left(p \in P(x) \rightarrow P(x) \cap d_{\alpha} \neq 0\right)\right\}
$$


This definition coincides with that of the closure of $d_{\alpha}$ in the usual topological sense $[\mathrm{Mun} 00]^{2}$

Lemma 3.3.4. Let $x \in S$ and suppose $U \subseteq P(x)$. Then there exists $\alpha<\omega_{1}$ such that

$$
d_{\alpha} \subseteq U \subseteq c_{\alpha}
$$

Proof. Letting $n=|x|$, we have the following inclusions:

$$
U \subseteq P(x) \subseteq F n_{<}(A B) \cap(A \times B)^{n} \subseteq \mathbb{R}^{2 n}
$$

Thus, there exists a countable $d \subseteq U$ which is dense in the relative topology on $U \subseteq \mathbb{R}^{2 n}$. Since $U \subseteq P(x)$, there exists $\alpha<\omega_{1}$ such that $d_{\alpha}=d \subseteq U$. On the other hand, if $\overline{d_{\alpha}}$ is the closure of $d_{\alpha}$ relative to $U$, we have $\overline{d_{\alpha}} \subseteq c_{\alpha}$, since $c_{\alpha}$ is the closure of $d_{\alpha}$ relative to $F n_{<}(A B) \cap(A \times B)^{n}$. Hence, $U=\overline{d_{\alpha}} \subseteq c_{\alpha}$.

\subsubsection{Exclusion}

For each $\alpha<\omega_{1}$, we now use the closures $c_{\beta}$ for $\beta<\alpha$ to exclude additional elements of $A$ and $B$ from the definitions of $A_{\alpha}$ and $B_{\alpha} \cdot{ }^{3}$ At each stage in the recursion indexed by $\langle\alpha, n\rangle$, we need the cumulative suborder

$$
\mathbb{P}_{\alpha n}=\left\{p \in F n_{<}(A B): p \text { respects the partition up through stage }\langle\alpha, n\rangle\right\}
$$

where the defining condition means that if $\langle a, b\rangle \in p \in \mathbb{P}_{\alpha n}$, then $\langle a, b\rangle=\left\langle k_{\beta m}, l_{\beta m^{\prime}}\right\rangle$ for some $\beta \leq \alpha$ and $m, m^{\prime} \leq n$. We note that $\mathbb{P}_{\alpha n}$ is a countable union of countable sets and therefore countable.

\footnotetext{
2 To see that $c_{\alpha}$ is the closure of $d_{\alpha}$, note that (i) the $p \in F n_{<}(A B)$ forming $c_{\alpha}$ are actually taken from $F n_{<}(A B) \cap(A \times B)^{n}$, where $n=\left|x_{\alpha}\right|$; and (ii) the collection $\left\{P(x) \cap(A \times B)^{n}: x \in S\right\}$ forms a basis for this space.

3 Recall that $A_{\alpha}=\left\{k_{\alpha n}: n<\omega\right\}$ and $B_{\alpha}=\left\{l_{\alpha n}: n<\omega\right\}$ where $\left\langle k_{\alpha n}, l_{\alpha n}\right\rangle$ are defined by recursion on $\omega$.
} 
Now define, for each $\beta<\alpha, m \leq n, i \in x_{\beta}$ and $p \in \mathbb{P}_{\alpha n}$, the "exclusion sets"

$$
\begin{aligned}
& X_{\beta m i p}=\left\{l \in B: p \cup\left\{\left\langle k_{\alpha m}, l\right\rangle\right\} \in c_{\beta} \text { and }\left\langle k_{\alpha m}, l\right\rangle \in i\right\} \\
& Y_{\beta m i p}=\left\{k \in A: p \cup\left\{\left\langle k, l_{\alpha m}\right\rangle\right\} \in c_{\beta} \text { and }\left\langle k, l_{\alpha m}\right\rangle \in i\right\},
\end{aligned}
$$

when $X_{\beta m i p}$ (resp., $Y_{\beta m i p}$ ) is countable; otherwise set $X_{m \beta i p}$ (resp., $Y_{m \beta i p}$ ) equal to 0. Intuitively, $X_{\beta m i p}$ consists of all $b \in B$ that would form an element $\langle a, b\rangle$ in the $i$-th rectangle of $x_{\beta}$, which when adjoined to some $p \in \mathbb{P}_{\alpha n}$, would form an element of $c_{\beta}$.

Finally, in place of our earlier selection of $l_{\alpha n}$ (resp., $k_{\alpha n}$ ) (see Section 3.2), we now exclude in addition any element in any $X_{\beta m i p}$ (resp., $Y_{\beta m i p}$ ). Specifically, if $k_{\alpha n}$ is the last-defined, select

$$
l_{\alpha n} \in\left(B \cap r_{n}\right)-\left(\bigcup_{\beta<\alpha} B_{\beta} \cup \bigcup_{\beta m i p} X_{\beta m i p}\right),
$$

and if $l_{\alpha n}$ is the last-defined, select

$$
k_{\alpha n+1} \in\left(A \cap r_{n+1}\right)-\left(\bigcup_{\beta<\alpha} A_{\beta} \cup \bigcup_{\beta m i p} Y_{\beta m i p}\right) \text {. }
$$

Since by definition each $X_{\beta m i p}$ and $Y_{\beta m i p}$ is countable, so are their unions; thus our earlier argument that such selections are possible remains valid. In addition, because the back-and-forth and diagonalization methods are applied simultaneously, we still have $A=\bigcup A_{\alpha}$ and $B=\bigcup B_{\beta}$ (Lemma 3.2.1).

\subsection{Definition of $\mathbb{P}(A B)$}

We now make

\section{Definition 3.4.1.}

$\mathbb{P}(A B)=\left\{p \in F n_{<}(A B): \forall \alpha<\omega_{1} \forall a \in A\left[a \in \operatorname{dom}(p) \rightarrow\left(a \in A_{\alpha} \leftrightarrow p(a) \in B_{\alpha}\right)\right]\right\}$ 
As we intended, $\mathbb{P}(A B)$ consists of all elements $p$ in $F n_{<}(A B)$ that respect the partitions of $A$ and $B$, i.e., if $\langle a, b\rangle \in p \in \mathbb{P}(A B)$, then $\langle a, b\rangle=\left\langle k_{\alpha m}, l_{\alpha m^{\prime}}\right\rangle$ for some $\alpha<\omega_{1}$ and $m, m^{\prime}<\omega$.

\section{5 $\mathbb{P}(A B)$ satisfies c.c.c.}

In this section we will show

Theorem 3.5.1. $\mathbb{P}(A B)$ satisfies the countable chain condition.

Proof. Suppose to the contrary there exists an uncountable antichain $U \subseteq \mathbb{P}(A B)$. The proof will be given in a series of lemmas.

Lemma 3.5.2. If there exists an uncountable antichain $U \subseteq \mathbb{P}(A B)$, then there exists an uncountable antichain $U^{\prime} \subseteq \mathbb{P}(A B)$ with the following properties:

(i) For all $p \in U^{\prime},|p|=n$ for some $n<\omega$, and $n$ is the least number with this property among all antichains in $\mathbb{P}(A B)$.

(ii) For all $p_{1}, p_{2} \in U^{\prime}, \operatorname{dom}\left(p_{1}\right) \cap \operatorname{dom}\left(p_{2}\right)=0=\operatorname{rng}\left(p_{1}\right) \cap r n g\left(p_{2}\right) .{ }^{4}$

(iii) $U^{\prime} \subseteq P(x)$ for some $x \in S$.

(iv) There exists $\alpha<\omega_{1}$, and a rectangle $i_{0} \in x_{\alpha}$, such that

$$
d_{\alpha} \subseteq U^{\prime} \subseteq c_{\alpha}
$$

and for every $p \in U^{\prime}$, if $\langle a, b\rangle$ is the last-constructed element of $p$, then $\langle a, b\rangle$ lies in $i_{0}$, and (uniformly over $U^{\prime}$ ) either $b$ is constructed after $a$, or $a$ is constructed after $b$.

Proof. We use throughout the pigeonhole principle (Lemma 2.2.1): if an uncountable set can be expressed as a countable union of sets, then at least one of the sets of the union must be uncountable.

\footnotetext{
${ }^{4}$ In this case, we will say that $U^{\prime}$ is pairwise disjoint.
} 
(i) Let $\mathcal{U}=\{U: U$ is an uncountable antichain in $\mathbb{P}(A B)\}$; by assumption, $\mathcal{U}$ is not empty. Each $U \in \mathcal{U}$ can be partitioned according to the finite cardinality of its elements. By the pigeonhole principle there is a least $n^{\prime}=n^{\prime}(U)$ such that $\left\{p \in U:|p|=n^{\prime}\right\}$ is uncountable. Let $n=\min \left\{n^{\prime}(U): U \in \mathcal{U}\right\}$, and let $U^{\prime}$ be an uncountable antichain with $|p|=n$ for all $p \in U^{\prime}$. Then $U^{\prime}$ has property $(i)$.

(ii) Assume $U$ has property $(i)$ and define $U^{\prime}=\left\{p_{\alpha}: \alpha<\omega_{1}\right\} \subseteq U$ by recursion as follows. Suppose $p_{\beta}$ has been defined for all $\beta<\alpha$, and choose

$$
\begin{aligned}
p_{\alpha} \in U-\left\{p \in U: \exists a \in \operatorname{dom}(p)\left(a \in \bigcup_{\beta<\alpha} \operatorname{dom}\left(p_{\beta}\right)\right) \vee\right. \\
\left.\qquad \exists b \in \operatorname{rng}(p)\left(b \in \bigcup_{\beta<\alpha} \operatorname{rng}\left(p_{\beta}\right)\right)\right\} .
\end{aligned}
$$

This definition makes sense, since for each of the countably many $a \in \bigcup_{\beta<\alpha} \operatorname{dom}\left(p_{\beta}\right)$, only countably many $b \in B$ exist such that $\langle a, b\rangle$ lies in some $p \in U$; similarly countably many $a \in A$ exist for each $b \in \bigcup_{\beta<\alpha} \operatorname{rng}\left(p_{\beta}\right)$; furthermore, each $\langle a, b\rangle$ occurs in only countably many elements of $U$ (otherwise, $U^{\prime}=\{p-\{\langle a, b\rangle\}:\langle a, b\rangle \in p \in U\}$ is an uncountable antichain of $\mathbb{P}(A B)$ of cardinality $n-1$, contradicting $(i)$ ). Thus, it follows that the set of $p \in U$ whose domain contains some $a \in \bigcup_{\beta<\alpha} \operatorname{dom}\left(p_{\beta}\right)$ or whose range contains some $b \in \bigcup_{\beta<\alpha} \operatorname{rng}\left(p_{\beta}\right)$ is only countable, so such a choice for $p_{\alpha}$ is in fact possible. It is now clear that $U^{\prime}$ satisfies property $(i i)$.

(iii) Since $\bigcup_{x \in S} P(x)=F n_{<}(A B)$, we can write $U=\bigcup_{x \in S}[U \cap P(x)]$. Since $U$ is uncountable but $S$ is countable, by the pigeonhole principle there is an $x \in S$ such that $U^{\prime}=U \cap P(x) \subseteq P(x)$ is uncountable.

(iv) Assume $U^{\prime}$ already has properties $(i),(i i)$, and (iii). Fix $x$ and $n$ satisfying $U \subseteq P(x)$ with $x \in S$ and $|x|=n$. Since each element of $U^{\prime}$ consists of $n$ pairs of reals, $U^{\prime}$ is essentially a subset of $\mathbb{R}^{2 n}$. Since $\mathbb{R}^{2 n}$ is separable, $U^{\prime}$ contains a 
countable subset $d$ which is dense in the relative topology on $U^{\prime}$. Recalling from Section 3.3.2 the enumeration of all countable sets in all $P(x)$, there exists $\alpha<\omega_{1}$ such that $d=d_{\alpha}$, and by Lemma 3.3.4, we have $d_{\alpha} \subseteq U^{\prime} \subseteq c_{\alpha}$.

Each $p \in U^{\prime}$ has a last-constructed element $\langle a, b\rangle$, and each such $\langle a, b\rangle$ lies in one of the $n$ rectangles of $x_{\alpha}$. By the pigeonhole principle, there exists a rectangle $i_{0} \in x_{\alpha}$ that contains an uncountable number of these last-constructed elements. Let $U^{\prime \prime}=\left\{p \in U\right.$ : the last-constructed element of $p$ lies in $\left.i_{0}\right\}$.

Again, if $\langle a, b\rangle \in p$ is the last-constructed element, then either $a$ or $b$ is last-constructed, and so we may assume that for an uncountable number of $p, b$ is constructed after $a$ (the argument that follows is symmetric in $a$ and $b$ ). Reverting to earlier notation, let $U^{\prime}$ stand for all $p$ in $U^{\prime \prime}$ for which the second coordinate of the last-constructed element is constructed last. Then $U^{\prime}$ has property $(i v)$.

Proof of Theorem 3.5.1 (continued). Without loss of generality we may now assume that $U$ has properties $(i),(i i),(i i i)$, (iv) above. Indeed, since Lemma 3.5.2 shows that if $U$ exists, then $U^{\prime}$ exists, any contradiction obtained assuming the existence of $U^{\prime}$ serves to contradict the existence of $U$. Accordingly, let $n<\omega$ satisfy property $(i)$, and fix $\alpha<\omega_{1}, i_{0} \in x_{\alpha}$ satisfying properties (iii) and (iv).

We will return to the proof of Theorem 3.5.1 after introducing certain auxiliary sets (namely, $Q, R, L$ below) and associated lemmas. To provide some motivation, we will at the same time preview the use of these sets.

In order to obtain a contradiction, we intend to construct distinct compatible elements $p_{1}, p_{2}$ in $U$. To this end, consider the set $Q$ of restrictions of elements in $U$ to all but their last-constructed element:

$$
Q=\left\{q \in F n_{<}(A B): q=p-i_{0} \wedge p \in U\right\}
$$

Assume $n>1$ (the case $n=1$ is handled separately). By minimality of $n=\left|x_{\alpha}\right|, Q$ 
is not an antichain in $\mathbb{P}(A B)$. Our strategy will be to choose distinct compatible $q_{1}, q_{2} \in Q$, find suitable $a_{1}, a_{2}, b_{1}, b_{2}$, and finally set $p_{1}=q_{1} \cup\left\{\left\langle a_{1}, b_{1}\right\rangle\right\}$ and $p_{2}=q_{2} \cup\left\{\left\langle a_{2}, b_{2}\right\rangle\right\}$. "Suitable" in this context means that $\left\{\left\langle a_{1}, b_{1}\right\rangle\right\}$ and $\left\{\left\langle a_{2}, b_{2}\right\rangle\right\}$ are compatible, i.e., $a_{1}<a_{2} \leftrightarrow b_{1}<b_{2}$.

To obtain the strict ordering, we consider

$$
A^{\prime}=A-\bigcup\left\{\operatorname{dom}(p): p \in d_{\alpha}\right\}
$$

Since $d_{\alpha}$ is countable, $A^{\prime}$ is uncountable. $A^{\prime}$ is used in conjunction with the sets of right $(R)$ and left $(L)$ "projected" limit points of $d_{\alpha}$, defined as follows. For any $p \in U$, let $a(p)$ satisfy $p \cap i_{0}=\{\langle a(p), b(p)\rangle\}$. Let

$$
\begin{aligned}
& R=\left\{p \in c_{\alpha}: \forall x \in S[p \in P(x) \rightarrow\right. \\
& \left.\left.\quad \exists p^{\prime} \in d_{\alpha} \cap P(x) \exists a^{\prime} \in A \exists b^{\prime} \in B\left(p^{\prime} \cap i_{0}=\left\{\left\langle a^{\prime}, b^{\prime}\right\rangle\right\} \wedge a^{\prime}<a(p) .\right)\right]\right\}, \\
& L=\left\{p \in c_{\alpha}: \forall x \in S[p \in P(x) \rightarrow\right. \\
& \left.\left.\quad \exists p^{\prime} \in d_{\alpha} \cap P(x) \exists a^{\prime} \in A \exists b^{\prime} \in B\left(p^{\prime} \cap i_{0}=\left\{\left\langle a^{\prime}, b^{\prime}\right\rangle\right\} \wedge a^{\prime}>a(p) .\right)\right]\right\} .
\end{aligned}
$$

The following lemma will then guarantee that we can find compatible $\left\langle a_{1}, b_{1}\right\rangle$, $\left\langle a_{2}, b_{2}\right\rangle$ in $i_{0}$.

Lemma 3.5.3. If $p \in c_{\alpha}$ is such that $p \cap i_{0}=\{\langle a, b\rangle\}$ and $a \in A^{\prime}$, then $p$ is either in $R$ or $L$, or both.

Proof. Suppose $p \notin R$. Then for some $x \in S$ such that $p \in P(x)$, all $p^{\prime} \in d_{\alpha} \cap P(x)$ have the property that if $p^{\prime} \cap i_{0}=\left\{\left\langle a^{\prime}, b^{\prime}\right\rangle\right\}$ then $a^{\prime} \geq a$. But $a \in A^{\prime}$ implies $a \notin \operatorname{dom}\left(p^{\prime}\right)$, so $a^{\prime}>a$ and thus $p \in L$.

The following lemma is basic. 
Lemma 3.5.4. (i) If $n=1$, then for all $a \in A^{\prime}$ the set $\left\{b \in B:\{\langle a, b\rangle\} \in c_{\alpha}\right\}$ is countable. (ii) If $n>1$, then there are uncountably many $q \in Q$ such that for all $a \in A^{\prime}$, the set $\left\{b \in B: p=q \cup\{\langle a, b\rangle\} \in c_{\alpha}\right\}$ is countable.

Proof. (i) Suppose not. Then for some $a \in A^{\prime}$, the set $\left\{b \in B:\{\langle a, b\rangle\} \in c_{\alpha}\right\}$ is uncountable; this gives us an uncountable set of $p \in c_{\alpha}$ each of which is in either in $R$ or $L$ (Lemma 3.5.3). Then by the pigeonhole principle, either $R$ or $L$ is uncountable. Assume $R$ is uncountable; a similar argument applies if $L$ is uncountable. Since $|R| \geq 2$, there exist distinct $\left\{\left\langle a, b_{1}\right\rangle\right\},\left\{\left\langle a, b_{2}\right\rangle\right\}$ in $R$ and disjoint rational intervals $s_{1}, s_{2}$ such that $b_{1} \in s_{1}, b_{2} \in s_{2}$, as well as a rational interval $r$ such that $a \in r$.

Assume $b_{1}<b_{2}$; otherwise, switch subscripts 1 and 2 in what follows. Let $x \in S$ be such that $x \cap i_{0}=r \times s_{1}$. Since $\left\{\left\langle a, b_{1}\right\rangle\right\} \in R$, there exists a $p_{1}^{\prime}=\left\{\left\langle a_{1}^{\prime}, b_{1}^{\prime}\right\rangle\right\} \in d_{\alpha} \cap P(x)$ with $a_{1}^{\prime}<a$. Since $p_{1}^{\prime} \in d_{\alpha} \cap\left(r_{1} \times s_{1}\right) \subseteq U \cap i_{0}=U$, $p_{1}^{\prime} \in U$. Now let $r^{\prime}$ be a rational interval with $a \in r^{\prime}$ but $a_{1}^{\prime} \notin r^{\prime}$. Since $\left\{\left\langle a, b_{2}\right\rangle\right\} \in R$, there exists $p_{2}^{\prime}=\left\{\left\langle a_{2}^{\prime}, b_{2}^{\prime}\right\rangle\right\} \in d_{\alpha} \cap\left(r^{\prime} \times s_{2}\right)$ with $a_{2}^{\prime}<a$. As above, $p_{2}^{\prime} \in U$. But by construction, $a_{1}^{\prime}<a_{2}^{\prime}$ and $b_{1}^{\prime}<b_{2}^{\prime}$. Thus $p_{1}^{\prime} \cup p_{2}^{\prime} \in \mathbb{P}(A B)$ is order-preserving, so $p_{1}^{\prime}, p_{2}^{\prime} \in U$ are compatible, a contradiction. ${ }^{5}$

(ii) If not, then only countably many $q \in Q$ have the stated property, which implies that uncountably many $q$ have the complementary property, namely, that for some $a_{q} \in A^{\prime}$ the set $\left\{b \in B: p=q \cup\left\{\left\langle a_{q}, b\right\rangle\right\} \in c_{\alpha}\right\}$ is uncountable. As in part (i), for each such $q$ we may pick $b_{q 1}, b_{q 2}$ such that if $p_{1}=q \cup\left\{\left\langle a_{q}, b_{q 1}\right\rangle\right\}$ and $p_{2}=q \cup\left\{\left\langle a_{q}, b_{q 2}\right\rangle\right\}$, then both $p_{1}, p_{2}$ lie in $R$.

Furthermore, setting $i_{0}=r_{0} \times s_{0}$, since the set of rational intervals is

\footnotetext{
${ }^{5}$ Both $p_{1}$ and $p_{2}$ must be taken from either $R$ or $L$ : for if $p_{1}=\left\{\left\langle a, b_{1}\right\rangle\right\} \in R$ and $p_{2}=\left\{\left\langle a, b_{2}\right\rangle\right\} \in$ $L$, then for a contradiction we would need $b_{1}<b_{2}$; however, nothing guarantees such a choice is possible.
} 
countable, we can pass again to an uncountable subset $Q^{\prime} \subseteq Q$ and find disjoint rational intervals $s, t \subseteq s_{0}$, such that $b_{q 1} \in s$ and $b_{q 2} \in t$ for all $q \in Q^{\prime}$. As before, assume $b_{q 1}<b_{q 2}$ (otherwise, switch 1 and 2 in what follows).

Since $Q^{\prime}$ is uncountable and $|q|=n-1$ for all $q \in Q^{\prime}$, by minimality of $n, Q^{\prime}$ is not an antichain in $\mathbb{P}(A B)$. Choose distinct compatible $q_{1}, q_{2} \in Q^{\prime}$ with $a_{q_{1}}<a_{q_{2}}$. Since $U$, and hence $q_{1}, q_{2}$, is pairwise disjoint, there are $x^{1}, x^{2} \in S$ so that $q_{1} \in P\left(x^{1}\right)$ and $q_{2} \in P\left(x^{2}\right)$ with all $i \in x^{1} \cup x^{2}$ pairwise separated and with each $i$ a subset of some $i^{\prime} \in x_{\alpha}$. By the separation property, it follows from the compatibility of $q_{1}, q_{2}$ that any element of $P\left(x^{1}\right)$ is compatible with any element of $P\left(x^{2}\right)$.

Now let $p_{1}=q_{1} \cup\left\{\left\langle a_{q_{1} 1}, b_{q 1}\right\rangle\right\} \in c_{\alpha}$ and $p_{2}=q_{2} \cup\left\{\left\langle a_{q^{2}}, b_{q^{2}}\right\rangle\right\} \in c_{\alpha}$. Since both $p_{1}, p_{2} \in R$, we can repeat the procedure in part $(i)$ to find $\left\langle a_{1}^{\prime}, b_{1}^{\prime}\right\rangle \in i_{0}$ and $q_{1}^{\prime} \in P\left(x^{1}\right)$ so that $p_{1}^{\prime}=q_{1}^{\prime} \cup\left\{\left\langle a_{1}^{\prime}, b_{1}^{\prime}\right\rangle\right\} \in d_{\alpha} \cap P\left(x_{\alpha}\right)$, and also $\left\langle a_{2}^{\prime}, b_{2}^{\prime}\right\rangle \in i_{0}$ and $q_{2}^{\prime} \in P\left(x^{2}\right)$ so that $p_{2}^{\prime}=q_{2}^{\prime} \cup\left\{\left\langle a_{2}^{\prime}, b_{2}^{\prime}\right\rangle\right\}$ is in $d_{\alpha} \cap P\left(x_{\alpha}\right)$, and $a_{1}^{\prime}<a_{2}^{\prime}$. But since $b_{1}^{\prime}<b_{2}^{\prime}$ and $q_{1}^{\prime}, q_{2}^{\prime}$ are compatible, we have that $p_{1}^{\prime}, p_{2}^{\prime} \in d_{\alpha} \subseteq U$ are compatible, a contradiction.

Lemma 3.5.5. There exists $q \in Q$ with the properties: (i) $\forall a^{\prime} \in A^{\prime}$ $\left\{b \in B: p=q \cup\left\{\left\langle a^{\prime}, b\right\rangle\right\} \in c_{\alpha}\right\}$ is countable. (ii) $\exists a \in A^{\prime} \exists b \in B$ such that $p=q \cup\{\langle a, b\rangle\} \in U$ and $\langle a, b\rangle$ is constructed after stage $\alpha$.

Proof. If $n=1$, let $q=0 \in Q$. By Lemma 3.5.4, $q$ satisfies property $(i)$. Since $U$ is uncountable and $U$ is pairwise disjoint, the sets $\left\{a \in A^{\prime}: \exists b \in B\{\langle a, b\rangle\} \in U\right\}$ and $\left\{b \in B: \exists a \in A^{\prime}\{\langle a, b\rangle\} \in U\right\}$ are also uncountable. Since $\bigcup_{\beta<\alpha} A_{\beta}$ and $\bigcup_{\beta<\alpha} B_{\beta}$ are only countable, some $p=\{\langle a, b\rangle\} \in U$ must be constructed after stage $\alpha$. Thus $q$ satisfies property $(i i)$.

If $n>1$, then by Lemma 3.5.4 (ii), uncountably many $q \in Q$ satisfy property (i). Again, because $U$, and thus $Q$, is disjoint, $\bigcup_{q \in Q} \operatorname{dom}(q)$ and $\bigcup_{q \in Q} \operatorname{rng}(q)$ are 
also both uncountable. Thus, as before some $q$ must contain an element $\langle r, s\rangle$ constructed after stage $\alpha$. By definition of $Q$, there exist $a \in A^{\prime}$ and $b \in B$ such that $p=q \cup\{\langle a, b\rangle\} \in U$. Since $\langle a, b\rangle$ is constructed after $\langle r, s\rangle, q$ satisfies property (ii).

Proof of Theorem 3.5 .1 (concluded). Fix $q \in Q, a \in A^{\prime}$, and $b \in B$ satisfying part (ii) of the previous Lemma. Then in part $(i)$ take $a^{\prime}=a$. This gives a $p \in c_{\alpha}$ containing $\langle a, b\rangle \in i_{0}$, where $b \in\left\{b^{\prime} \in B: q \cup\left\{\left\langle a, b^{\prime}\right\rangle\right\} \in c_{\alpha}\right\}$, and this latter set is countable. Moreover, $b$ is constructed at a stage $\beta>\alpha$ and after $a$. But this means that $b=l_{\beta m}$ belongs to the exclusion set $X_{\alpha m^{\prime} i_{0} q}$ for some $m, m^{\prime}, m^{\prime} \leq m$ (see Section 3.3.4). Since this contradicts the definition of $b, \mathbb{P}(A B)$ satisfies the countable chain condition.

\section{6 $\mathbb{P}(A B)$ forces $A \simeq B$}

Finally, we check that $\mathbb{P}(A B)$ forces an order-isomorphism between $A$ and $B$ in any generic extension of $M$ :

Theorem 3.6.1. $\quad 1_{\mathbb{P}(A B)} \Vdash A \simeq B$.

Proof. Consider $D_{a}=\{p \in \mathbb{P}(A B): a \in \operatorname{dom}(p)\}$ and $E_{b}=\{p \in \mathbb{P}(A B): b \in \operatorname{rng}(p)\}$ for fixed $a \in A, b \in B$. We first show that $D_{a}$ is a dense subset of $\mathbb{P}(A B)$ (see Section 2.3.1); the same is true of $E_{b}$ by a similar argument.

Suppose $q \in \mathbb{P}(A B)$; clearly, we may assume that $q \notin D_{a}$. Then for some $n<\omega$, we have

$$
q=\left\{\left\langle a_{1}, b_{1}\right\rangle, \ldots,\left\langle a_{n}, b_{n}\right\rangle\right\},
$$

where for all $m<n, a_{m} \neq a$, and for all $1 \leq i<j \leq n, a_{i}<a_{j} \leftrightarrow b_{i}<b_{j}$. Suppose $a \in A_{\alpha}$ and that $j, k$ are such that $a_{j}<a<a_{k}$. Since $B_{\alpha}$ is dense in $B$, we can find 
$b \in B_{\alpha}$ such that $b_{j}<b<b_{k}$. Let $p=q \cup\{\langle a, b\rangle\}$. Then $p \in D_{a}$ and for all $a, a^{\prime} \in \operatorname{dom}(p)\left(a<a^{\prime} \leftrightarrow p(a)<p\left(a^{\prime}\right)\right)$, so that $p \leq q$. Thus, $D_{a}$ (and similarly, $E_{b}$ ) is dense in $\mathbb{P}(A B)$.

If $G$ is $\mathbb{P}(A B)$-generic over $M$, then we claim that $f=\bigcup G$ is an order-isomorphism from $A$ to $B$ in $M[G]$. Indeed, that $f$ is bijective is clear since the $D_{a}$ and $E_{b}$ are dense. Furthermore, suppose $a, a^{\prime} \in A$ are such that $a<a^{\prime}$ but $f(a) \nless f\left(a^{\prime}\right)$; then there are $p_{1}, p_{2} \in G$ such that $a \in \operatorname{dom}\left(p_{1}\right)$ and $a^{\prime} \in \operatorname{dom}\left(p_{2}\right)$. But since $G$ is a filter, there exists $p \in G$ with $p \leq p_{1} \cup p_{2}$. Thus, we have $f(a)<f\left(a^{\prime}\right)$, a contradiction. 


\section{CHAPTER 4}

\section{ITERATED FORCING}

\subsection{Overview}

In this chapter we discuss the method of iterated forcing by which is constructed a model $M^{*}$ of set theory in which all $\aleph_{1}$-dense sets are isomorphic. This iteration may be seen as an additional outer recursion on $\omega_{2}$ defining in $M$ a partial order $\mathbb{P}$ forcing $M^{*}$, with each step based on some $\mathbb{P}(A B)$ as constructed in the last chapter. $\mathbb{P}=\mathbb{P}_{\omega_{2}}$ is the last in a sequence of c.c.c. partial orders $\left\langle\mathbb{P}_{\eta}\right\rangle$, $\eta \leq \omega_{2}$, called an iterated forcing structure. ${ }^{1}$ The following properties of $\left\langle\mathbb{P}_{\eta}\right\rangle$ convey the basic idea behind iterated forcing:

(1) There exists a sequence of extensions $\left\langle M_{\eta}\right\rangle$ ordered by inclusion:

$$
M=M_{0} \subseteq M_{1} \subseteq \cdots \subseteq M_{\eta} \subseteq \cdots \subseteq M_{\omega_{2}}=M^{*}
$$

such that each $\mathbb{P}_{\eta}$ forces $M_{\eta}$ over $M$.

(2) Every pair of $\aleph_{1}$-dense sets $A, B$ in $M^{*}$ appears in some $M_{\eta}$.

(3) $\mathbb{P}_{\eta+1}$ is defined in terms of some $\mathbb{P}(A B)$ in such a way that $\mathbb{P}_{\eta+1}$ is c.c.c. and forces $M_{\eta+1} \models A \simeq B$. From this, it follows that $M^{*} \models A \simeq B$.

\footnotetext{
${ }^{1}$ Such structures may be defined on any limit ordinal $\alpha$, but we will assume $\alpha=\omega_{2}$ and often leave the condition $\eta \leq \omega_{2}$ implicit.
} 
(4) The structure $\left\langle\mathbb{P}_{\eta}\right\rangle$ exhausts all $\aleph_{1}$-dense pairs $A, B \in M^{*}$, i.e., for each such $A, B$ there is an $\eta<\omega_{2}$ such that $A, B$ appear in $M_{\eta}$ and $\mathbb{P}_{\eta+1}$ is defined by $\mathbb{P}(A B)$ according to (3). This exhaustiveness property is particularly important since new $\aleph_{1}$-dense sets may appear at any stage of the iteration; it is here that the c.c.c. property of the $\mathbb{P}_{\eta}$ is used.

(5) (a) For $\eta \leq \omega_{2}$, the cardinals of all $M_{\eta}$ 's are the same; and (b) if $\mathrm{CH}$ and $\mathrm{CH}_{1}$ hold in $M$, then $\mathrm{CH}$ and $\mathrm{CH}_{1}$ hold in all $M_{\eta}$ with $\eta \leq \omega_{2}$, with the sole exception that in $M_{\omega_{2}}, 2^{\aleph_{0}}=\aleph_{2}$.

In what follows, we will focus on properties (1) and (4). The basic structure giving rise to (1) is the set of complete embeddings, discussed in the following section, which essentially order the $\mathbb{P}_{\eta}$ by forcing strength. For property (4), the basic structure is the bookkeeping function (Section 4.4). Property (2), that every $\aleph_{1}$-dense $A \in M^{*}$ appears in some $M_{\eta}$, will be discussed summarily in Section $4.3 .^{2}$

\subsection{Complete embeddings}

The structure $\left\langle\mathbb{P}_{\eta}\right\rangle$ forces a sequence of extensions $\left\langle M_{\eta}\right\rangle$ ordered by inclusion and with $M^{*}=M_{\omega_{2}}$. For this to be true, the $\mathbb{P}_{\eta}$ must themselves be ordered by forcing strength, i.e.,

$$
\mathbb{P}_{0} \subseteq_{c} \mathbb{P}_{1} \subseteq_{c} \cdots \subseteq_{c} \mathbb{P}_{\eta} \subseteq_{c} \cdots \subseteq_{c} \mathbb{P}_{\omega_{2}}=\mathbb{P}
$$

where the relation $\subseteq_{c}$ expresses that the forcing structure of each $\mathbb{P}_{\eta}$ "embeds" into its successors. More precisely, $\mathbb{P} \subseteq_{c} \mathbb{Q}$ if and only if there exists a complete

\footnotetext{
${ }^{2}$ For property $(3)$, in a rigorous presentation $\mathbb{P}_{\eta+1}$ is defined in terms of $\mathbb{P}_{\eta}$ and a $\mathbb{P}_{\eta}$-name for $\mathbb{P}(A B)$ (see Section 4.2), but the technicalities associated with the method of forcing tend to obscure the main ideas. Therefore, we will simply assume property (3); furthermore, little will be said about limit stages, except to mention the notion of finite support at limits, which is important for property (2) and crucial for proving that each $\mathbb{P}_{\eta}$ is in fact c.c.c. Property (5a) is a consequence of $\mathbb{P}_{\eta}$ being c.c.c. (see [Kun80]); the first part of $(5 \mathrm{~b})$ can be shown using nice names, and $2^{\aleph_{0}}=\aleph_{2}$ in $M_{\omega_{2}}$ is a consequence of Sierpinski's result mentioned on page 1.
} 
embedding $i: \mathbb{P} \rightarrow \mathbb{Q}$ where the latter term is defined as follows:

Definition 4.2.1. Suppose $\mathbb{P}=\left\langle\mathbb{P}, \leq_{\mathbb{P}}, 1_{\mathbb{P}}\right\rangle$ and $\mathbb{Q}=\left\langle\mathbb{Q}, \leq_{\mathbb{Q}}, 1_{\mathbb{Q}}\right\rangle$ are partial orders in $M$. A function $i: \mathbb{P} \rightarrow \mathbb{Q}$ is a complete embedding iff (i) $\forall p, p^{\prime} \in \mathbb{P}\left(p \leq_{\mathbb{P}} p^{\prime} \rightarrow i(p) \leq_{\mathbb{Q}} i\left(p^{\prime}\right)\right) ;$ (ii) $\forall p, p^{\prime} \in \mathbb{P}\left(p \perp p^{\prime} \leftrightarrow i(p) \perp i\left(p^{\prime}\right)\right)$; (iii) $\forall q \in \mathbb{Q} \exists p \in \mathbb{P} \forall p^{\prime} \in \mathbb{P}\left(p^{\prime} \leq p \rightarrow i\left(p^{\prime}\right)\right.$ and $q$ are compatible in $\left.\mathbb{Q}\right)$.

The idea is that the conditions (ii) and (iii) guarantee that $i$ preserves generic sets in the following sense:

Lemma 4.2.2. Suppose $i: \mathbb{P} \rightarrow \mathbb{Q}$ is a complete embedding and $H$ is $\mathbb{Q}$-generic over $M$. Then $G=\{p \in \mathbb{P}: i(p) \in H\}$ is $\mathbb{P}$-generic over $M$ and $M[G] \subseteq M[H]$.

To obtain a chain $\left\langle M_{\eta}\right\rangle$, we need complete embeddings $i_{\eta \xi}: \mathbb{P}_{\eta} \rightarrow \mathbb{P}_{\xi}$ for all $\eta<\xi \leq \omega_{2}$. To discuss these, we sketch some relevant features of the construction of $\left\langle\mathbb{P}_{\eta}\right\rangle$, which involves an auxiliary sequence $\left\langle\pi_{\eta}\right\rangle$, as follows:

(1) $\mathbb{P}_{0}=\{0\}$

(2) $\mathbb{P}_{\eta}$ is a partial order consisting of $\eta$-sequences of names for partial orders, and $\pi_{\eta}$ is a $\mathbb{P}_{\eta}$-name for a partial order $\mathbb{P}(A B)$.

(3) If $p \in \mathbb{P}_{\eta}$, then $p=\left\langle\rho_{\mu}: \mu<\eta\right\rangle$ and $\rho_{\mu} \in \operatorname{dom}\left(\pi_{\mu}\right)$ for all $\mu<\eta$.

(4) An $(\eta+1)$-sequence of names for partial orders belongs to $\mathbb{P}_{\eta+1}$ iff it extends some $\eta$-sequence in $\mathbb{P}_{\eta}$ by some $\rho \in \operatorname{dom}\left(\pi_{\eta}\right)$.

(5) Finite support, i.e., if $p \in \mathbb{P}_{\eta}$, then for all but finitely many $\mu<\eta$, $p \nmid \mu \Vdash p_{\mu}=1$.

Under this scheme, an iterated forcing structure $\left\langle\mathbb{P}_{\eta}\right\rangle$ is fully determined once $\left\langle\pi_{\eta}\right\rangle$ is specified; this, in turn, is carried out using a bookkeeping function discussed later. 
The complete embeddings $i_{\eta \xi}$ we need are now given by:

Definition 4.2.3. Suppose $\eta<\xi \leq \omega_{2}$ and $p=\left\langle\rho_{\mu}: \mu<\eta\right\rangle \in \mathbb{P}_{\eta}$. Then $i_{\eta \xi}(p)=\left\langle\rho_{\mu}: \mu<\eta\right\rangle \frown\left\langle 1_{\mu}: \eta \leq \mu<\xi\right\rangle \in \mathbb{P}_{\xi}$,

where the symbol $\frown$ denotes concatenation. The function $i$ "pads out" each element of $\mathbb{P}_{\eta}$ with $\xi-\eta$ many $1_{\mu}$ 's, where $1_{\mu}$ is strictly speaking a name for the largest element in $\mathbb{P}_{\mu}$. The complete embedding property follows from this definition, and Lemma 4.2.2 can now be extended as follows.

Assume $M^{*}=M[G]$ and that $G$ is $\mathbb{P}_{\omega_{2}}$-generic over $M$. For each $\eta<\omega_{2}$, define

$$
G_{\eta}=\left\{p \in \mathbb{P}_{\eta}: i_{\eta \omega_{2}}(p) \in G\right\}
$$

and set $M_{\eta}=M\left[G_{\eta}\right]$; then $\left\langle\mathbb{P}_{\eta}\right\rangle$ and $\left\langle M_{\eta}\right\rangle$ satisfy property (1) of Section 4.1.

\subsection{Appearance at intermediate stages}

For the method of iterated forcing to work, every pair of $\aleph_{1}$-dense sets in $M^{*}$ must appear in some intermediate $M_{\eta}$. But although the $\left\langle M_{\eta}\right\rangle$ forms a chain under inclusion, it is not true that $M^{*}=\bigcup_{\eta<\omega_{2}} M_{\eta}$. Nonetheless, every $\aleph_{1}$-dense set in $M^{*}$ does appear in some $M_{\eta}$; to see this, we need the following fact.

Lemma 4.3.1. Let $\left\langle\mathbb{P}_{\eta}: \eta \leq \omega_{2}\right\rangle$ be an iterated forcing structure with finite support with $M^{*}$ as above. If $X \in M^{*}$ and $X \subseteq S \in M$ where $|S|<\aleph_{2}$ in $M^{*}$, then for some $\eta<\omega_{2}, X \in M_{\eta}$.

Corollary 4.3.2. Every $\aleph_{1}$-dense set in $M^{*}$ appears in some $M_{\eta}, \eta<\omega_{2}$.

Proof. Let $A$ be $\aleph_{1}$-dense in $M^{*}$, and suppose $f: \omega_{1} \rightarrow \mathcal{P}(\omega)$ is a bijection onto $A$ in $M^{*}$ (we are identifying $\mathcal{P}(\omega)$ with $\mathbb{R}$ ). Let $X=\bigcup\left\{\{\alpha\} \times f(\alpha): \alpha<\omega_{1}\right\} \in M^{*}$.

Since $X \subseteq \omega_{1} \times \omega$, by the Lemma, $X \in M_{\eta}$ for some $\eta<\omega_{2}$. It follows that $A=\operatorname{rng}(f)$ is also in $M_{\eta}$. 


\subsection{Bookkeeping function}

Finally, we consider the property that $\left\langle\mathbb{P}_{\xi}\right\rangle$ is exhaustive, i.e., $\mathbb{P}_{\omega_{2}}$ forces isomorphisms between all $\aleph_{1}$-dense sets $A, B$ in $M^{*}$. To accomplish this, $\left\langle\mathbb{P}_{\xi}\right\rangle$ is constructed using a bookkeeping function $b$ defined on $\aleph_{2}$. The value $b(\xi)$ indicates names of the $\aleph_{1}$-dense sets $A, B \in M^{*}$ by which we define $\mathbb{P}_{\xi+1}$ using a name for $\mathbb{P}(A B)$. Exhaustiveness follows from two facts about the function $b$ : (i) every such $A, B$ have names in the range of $b$; and (ii) there exist ordinals $\eta$ and $\xi$ such that: (a) $\eta \leq \xi<\omega_{2}$; (b) $A, B$ appear in $M_{\eta}$; and (c) $b(\xi)$ is a name for the pair $A, B$.

First, we must revisit our ground model $M$. For such a $b$ to exist, we will need a collection of names for (at least) isomorphic copies of all $\aleph_{1}$-dense sets in each $M_{\eta}$, with the size of each collection at most $\aleph_{2}$. For this to be true, the number of such sets themselves must, of course, be $\leq \aleph_{2}$. We can guarantee this result by now assuming that $M$ satisfies $\mathrm{CH}_{1}$ as well as $\mathrm{CH}$.

Secondly, recall that Lemma 2.5.7 gives such a bound for the nice names for subsets in $M_{\eta}$ of $\aleph_{1} \times \aleph_{1}$. This is enough for our purposes, since if $A=\langle A,<\rangle$ is $\aleph_{1}$-dense in $M_{\eta}$, a bijection $f: A \rightarrow \aleph_{1}$ exists in $M_{\eta}$ by Lemma 2.3.2. Thus, $A$ is isomorphic to the linear order $\left\langle\aleph_{1}, \prec\right\rangle$, where $\prec \subseteq \aleph_{1} \times \aleph_{1}$ is the image of $<$ under $f \times f$. In the sequel, we sometimes refer to $\aleph_{1}$-dense sets when we actually mean subsets of $\aleph_{1} \times \aleph_{1}$. With this in hand, we can now establish the existence of a bookkeeping function.

Lemma 4.4.1. There exists a function $b: \aleph_{2} \rightarrow \aleph_{2} \times \aleph_{2} \times \aleph_{2}$ such that $b$ is onto and if $b(\xi)=\langle\eta, \gamma, \delta\rangle$, then $\eta \leq \xi$.

Proof. By Lemma 2.2.2, let $g: \aleph_{2} \rightarrow \aleph_{2} \times \aleph_{2} \times \aleph_{2} \times \aleph_{2}$ be onto. Given $\xi<\aleph_{2}$ and 
$g(\xi)=\langle\eta, \gamma, \delta, \rho\rangle$, define $b: \aleph_{2} \rightarrow \aleph_{2} \times \aleph_{2} \times \aleph_{2}$ by

$$
b(\xi)= \begin{cases}\langle\eta, \gamma, \delta\rangle & \text { if } \eta \leq \xi \\ \langle 0,0,0\rangle & \text { if } \eta>\xi\end{cases}
$$

Clearly, if $b(\xi)=\langle\eta, \gamma, \delta\rangle$ then $\eta \leq \xi$. To see that $b$ is onto, fix $\langle\eta, \gamma, \delta\rangle \in \aleph_{2} \times \aleph_{2} \times \aleph_{2}$ and consider the set $S=\left\{\xi<\aleph_{2}: \exists \rho<\aleph_{2}(g(\xi)=\langle\eta, \gamma, \delta, \rho\rangle)\right\}$. Since the cardinality of $S$ is $\aleph_{2}$, and $\aleph_{2}$ is a regular cardinal, $S$ is unbounded in $\aleph_{2}$. Thus there exists $\xi \geq \eta$ such $g(\xi)=\langle\eta, \gamma, \delta, \rho\rangle$, and so $b(\xi)=\langle\eta, \gamma, \delta\rangle$.

To construct $\left\langle\mathbb{P}_{\eta}\right\rangle$, a bookkeeping function $b$ as provided by Lemma 4.4.1 is first prescribed. $\mathbb{P}_{\xi+1}$ is built from $\mathbb{P}_{\xi}$ and a $\mathbb{P}_{\xi}$-name for a $\mathbb{P}(A B)$, where $A, B$ appear in $M_{\eta}$ for some $\eta \leq \xi$. Index $\eta$, and $A, B \in M_{\eta}$ are determined by the value $b(\xi)=\langle\eta, \gamma, \delta\rangle$; more precisely, $\gamma<\aleph_{2}$ indicates a $\mathbb{P}_{\eta^{-}}$name for $A$, and $\delta<\aleph_{2}$ a $\mathbb{P}_{\eta}$-name for $B$.

For $b$ to be exhaustive, all $\aleph_{1}$-dense sets in a given $M_{\eta}$ must have $\mathbb{P}_{\eta^{-} \text {-names }}$ enumerable by $\aleph_{2}$. But $\mathbb{P}_{\eta}$ satisfies the c.c.c., so that Lemma 2.5.7, giving just such a bound on the cardinality of nice $\mathbb{P}_{\eta}$-names, is applicable as soon as we confirm that $\left|\mathbb{P}_{\eta}\right|=\aleph_{1}$ for all $\eta<\omega_{2}$. This in fact follows by induction on the construction of $\mathbb{P}_{\eta+1}$ from $\mathbb{P}_{\eta}$; to see this, recall that an $(\eta+1)$-sequence of names belongs to $\mathbb{P}_{\eta+1}$ iff it extends some $\eta$-sequence in $\mathbb{P}_{\eta}$ by some $\rho \in \operatorname{dom}\left(\pi_{\eta}\right)$, where $\pi_{\eta}$ names some $\mathbb{P}(A B)$; now observe that $\left|\rho \in \operatorname{dom}\left(\pi_{\eta}\right)\right|=|\mathbb{P}(A B)| \leq\left|F n_{<}(A B)\right|=\aleph_{1}$.

Lastly, we need a function $i_{\eta \xi}^{*}$ in $M$ from the set of nice $\mathbb{P}_{\eta}$-names to the set of

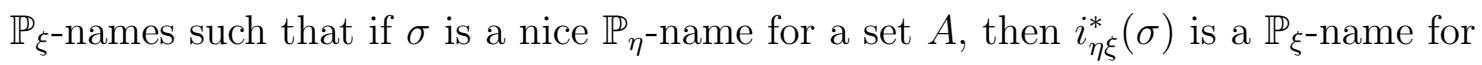
the same set $A$. In fact, such a function may be defined in terms of the complete embedding $i_{\eta \xi}$ discussed in Section 4.2; we omit the details. Then, if $b(\xi)=\langle\eta, \gamma, \delta\rangle$, 


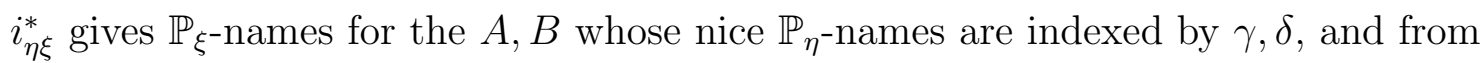

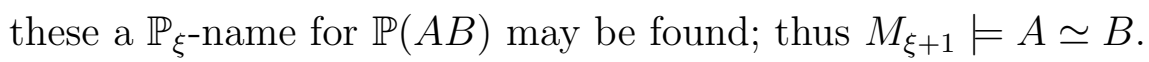

\subsection{Summary}

Collecting our results, we can finally show Baumgartner's result $(*)$ :

Theorem 4.5.1. If there exists a model of set theory, then there exists a model of set theory in which all $\aleph_{1}$-dense sets are isomorphic.

Proof. If there exists a model of ZFC, let $M$ be a countable transitive model of ZFC $+\mathrm{CH}+\mathrm{CH}_{1}$. Let $\left\langle\mathbb{P}_{\eta}, \eta \leq \omega_{2}\right\rangle$ be an iterated forcing structure in $M$, and let $M^{*}$ be a generic extension by $P_{\omega_{2}}$ over $M$. If $A, B \in M^{*}$ are $\aleph_{1}$-dense, then they appear in some $M_{\eta}$ and are isomorphic to subsets of $\aleph_{1} \times \aleph_{1}$ (also denoted by $A, B$ ). These $A, B$ have nice names with indices in the range of the bookkeeping function $b$. Since $b$ is onto, there exists $\xi \geq \eta$ such that $\mathbb{P}_{\xi+1}$ forces $M_{\xi+1} \models A \simeq B$, and hence $M^{*} \models A \simeq B$. 


\section{CHAPTER 5}

\section{CONCLUSION}

Using iterated forcing in ZFC, we have argued with some effort that if there exists any model of set theory at all, then there exists a model of set theory in which all $\aleph_{1}$-dense sets are isomorphic. It happens there are other interesting results derivable by similar methods, and the question arises whether there exists some general and natural principle of forcing of which these various results are simpler consequences.

In fact, the Proper Forcing Axiom (PFA) is such a principle, though it is not usually presented in its most natural form (see below). PFA applies to forcing by proper partial orders, and to avoid entering into an extended discussion, we merely note that all c.c.c. partial orders are proper, ${ }^{1}$ and that compositions of proper orders are proper. A natural formulation of the Proper Forcing Axiom is as follows [Bag99]:

Let $M$ be a transitive model of set theory, and let $\mathcal{A}, \mathcal{B} \in M$ be any two structures with $|\mathcal{A}|=\aleph_{1}$. If in a forcing extension over $M$ by a proper partial order $\mathbb{P}$ there exists an isomorphism of $\mathcal{A}$ onto a substructure of $\mathcal{B}$, then such an isomorphism already exists in $M$.

We conclude by showing that use of PFA avoids the technicalities appearing (and not appearing) in Chapter 4 related to infinite-stage iterated forcing. In fact,

\footnotetext{
${ }^{1}$ So PFA is a strengthening of Martin's Axiom $\left(\aleph_{1}\right)$.
} 
suppose that PFA holds and that $M$ is a countable transitive model of ZFC. If for any $A, B \aleph_{1}$-dense in $M$, we can find a proper forcing extension $M(A B)$ in which $A \simeq B$, then PFA will give us directly that $A \simeq B$ in $M$. The important thing is that we can obtain $M(A B)$ with only a two-stage iterated proper forcing.

Theorem 5.0.2. (PFA) In any model $M$ of set theory, all $\aleph_{1}$-dense sets are isomorphic.

Proof. Suppose $A, B$ are $\aleph_{1}$-dense in $M$. Stage 1: Force over $M$ using $Q=$ the set of countable partial functions from $\aleph_{1}$ to $2^{\aleph_{0}}$ ordered by reverse inclusion. $Q$ (it may be shown) is proper and collapses $2^{\aleph_{0}}$ onto $\aleph_{1}$, so that $\mathrm{CH}$ is true in every generic extension $M^{Q}$ of $M$ by $Q$. Stage 2 : Since $M^{Q}$ satisfies $\mathrm{CH}$, there exists a c.c.c. partial order $\mathbb{P}(A B)$ in $M^{Q}$ as found in Chapter 3. If we let $M(A B)=M^{Q \circ \mathbb{P}(A B)}$, where $Q \circ \mathbb{P}(A B)$ denotes composition of $Q$ and $\mathbb{P}(A B)$, then $A \simeq B$ in $M(A B)$. Since $Q \circ \mathbb{P}(A B)$ is proper, by $\mathrm{PFA}$ we obtain $A \simeq B$ in $M$. 


\section{BIBLIOGRAPHY}

[Bag99] J. Bagaria, Generic absoluteness and forcing axioms, in Models, algebras, and proofs (Bogotá, 1995): 1-12, Dekker, 1999.

[Baum73] J. E. Baumgartner, All $\aleph_{1}$-dense sets of reals can be isomorphic, Fundamenta Mathematicae, 79(2):101-106, 1973.

[Baum84] J. E. Baumgartner, Applications of the Proper Forcing Axiom, in Handbook of Set Theoretic Topology, ed. K. Kunen, J. E. Vaughan, North-Holland, 1984.

[Jech03] T. Jech, Set theory, Springer, 2003.

[Kun80] K. Kunen, Set theory, an introduction to independence proofs, Elsevier, 1980.

[Mo06] J. T. Moore, A five element basis for the uncountable linear orders, Annals of Mathematics (2), 163(2):669-688, 2006.

[Mun00] James R. Munkres, Topology, 2nd ed., Prentice Hall, 2000.

[Tod98] S. Todorčević, Basis problems in combinatorial set theory, in Proceedings of the International Congress of Mathematicians, Vol. II (Berlin, 1998), Extra Vol. II, 43:52, 1998. 